\title{
Kinetic theory for massive spin-1/2 particles from the Wigner-function formalism
}

\author{
Nora Weickgenannt, ${ }^{1}$ Xin-li Sheng, ${ }^{1,2}$ Enrico Speranza, ${ }^{1}$ Qun Wang, ${ }^{2}$ and Dirk H. Rischke ${ }^{1,2}$ \\ ${ }^{1}$ Institute for Theoretical Physics, Goethe University, Max-von-Laue-Strasse 1, \\ D-60438 Frankfurt am Main, Germany \\ ${ }^{2}$ Interdisciplinary Center for Theoretical Study and Department of Modern Physics, \\ University of Science and Technology of China, Hefei, Anhui 230026, China
}

(Received 27 February 2019; revised manuscript received 16 August 2019; published 25 September 2019)

\begin{abstract}
We calculate the Wigner function for massive spin-1/2 particles in an inhomogeneous electromagnetic field to leading order in the Planck constant $\hbar$. Going beyond leading order in $\hbar$ we then derive a generalized Boltzmann equation in which the force exerted by an inhomogeneous electromagnetic field on the particle dipole moment arises naturally. Furthermore, a kinetic equation for this dipole moment is derived. Carefully taking the massless limit we find agreement with previous results. The case of global equilibrium with rotation is also studied. Finally, we outline the derivation of fluid-dynamical equations from the components of the Wigner function. The conservation of total angular momentum is promoted as an additional fluid-dynamical equation of motion. Our framework can be used to study polarization effects induced by vorticity and magnetic field in relativistic heavy-ion collisions.
\end{abstract}

DOI: 10.1103/PhysRevD.100.056018

\section{INTRODUCTION}

Relativistic heavy-ion collisions (HICs) create a new phase of hot and dense strong-interaction matter, the quarkgluon plasma (QGP) (see e.g., Ref. [1]). The interaction rates between its constituents are sufficiently large that the matter rapidly reaches a state which can be described by fluid dynamics [2]. In noncentral HICs the global angular momentum generates a nonvanishing vorticity of the QGP fluid. Furthermore, in such collisions a strong magnetic field is formed due to the electric current produced by the spectator protons constituting the colliding ions.

In the QGP, quarks can be considered as (nearly) massless fermions. The interplay between the chiral anomaly on the one hand and the magnetic field and the fluid vorticity on the other hand gives rise to novel transport phenomena called chiral effects. Two such phenomena are the chiral magnetic effect (CME) [3] and the chiral vortical effect (CVE) [4], where a charge current is induced along the direction of the magnetic field and the vorticity, respectively. Large-scale experimental efforts are currently under way to discover these phenomena in HICs (for a recent review, see Ref. [5]).

From the theoretical point of view, it is therefore mandatory to develop a theory which allows to study such

Published by the American Physical Society under the terms of the Creative Commons Attribution 4.0 International license. Further distribution of this work must maintain attribution to the author(s) and the published article's title, journal citation, and DOI. Funded by SCOAP ${ }^{3}$. transport phenomena in chiral fluids. One approach is chiral kinetic theory, which has been derived using various methods, e.g., the classical action [6-14], the Wigner function [15-20], and the world-line formalism [21-23]. In Refs. $[15,16]$ it was shown that, using Wigner functions, one is able to recover the "side-jump" phenomenon first discussed in Refs. [11,13] in order to ensure total angularmomentum conservation in binary collisions. Furthermore, the inclusion of the chiral effects in fluid dynamics was studied in Refs. [4,24,25].

Another intriguing phenomenon occurring in the rotating QGP is that particles in the medium can be polarized in a way resembling the Einstein-de Haas [26] and Barnett effects [27]. Recently, the STAR Collaboration presented experimental evidence for the alignment of the spin of $\Lambda$ hyperons with the global angular momentum in peripheral HICs [28]. This finding revealed, for the first time, the strong vortical structure of the QGP. Many theoretical works have explored spin-polarization mechanisms triggered by vorticity in HICs. In particular, the importance of the spin-orbit interaction [29-31] and the relation between spin polarization and thermal vorticity in local thermodynamical equilibrium have been studied [32-35]. A fluid-dynamical description, which includes the space-time evolution of the spin polarization, was proposed in Refs. [36-39]. However, this formulation is based on a specific choice for the energy-momentum and spin tensors. The physical implications of different sets of energy-momentum and spin tensors in fluid dynamics was investigated in Ref. [40].

Although there has been intense theoretical activity which has led to a deeper understanding of the transport 
properties of chiral matter, few studies have attempted to derive a covariant kinetic theory for massive particles using Wigner functions [41,42]. The aim of this paper is to fill this gap. We derive kinetic theory for massive spin- $1 / 2$ particles in an inhomogeneous electromagnetic field as a basis to study polarization effects in HICs. Our starting point is the covariant formulation of the Wigner function [43-49]. In order to solve the equations of motion for the Wigner function, we employ an expansion in the Planck constant $\hbar$ and truncate at the lowest nontrivial order. This approximation is valid if the following two assumptions hold:

(i) $\hbar\left|\gamma^{\mu} \nabla_{\mu} W\right| \ll m|W|$, where $W$ is the Wigner function, $m$ is the particle mass, $\nabla_{\mu}$ represents the gradient operator in Eq. (6) $[45,50]$, and the modulus applies to each component of the corresponding matrix in Dirac space,

(ii) $\hbar \ll \Delta R \Delta P$, where $\Delta R$ is a spatial scale over which the electromagnetic field tensor varies significantly and $\Delta P$ a momentum scale over which the Wigner function varies significantly.

Assumption (i) implies that the $\hbar$-expansion is effectively a gradient expansion. Assumption (ii) allows us to truncate the power-series expansion of the Bessel functions entering the equations of motion of the Wigner function [45].

Under these assumptions, we first give an explicit derivation of the leading-order solution. Then, considering the equation of motion for the Wigner function to first and second order in $\hbar$, we derive a generalized Boltzmann equation, where the external force acting on the particles is given by two contributions. The first one is the Lorentz force, which gives rise to the usual Vlasov term, and the second one is the Mathisson force [51], i.e., the force exerted on the particle's dipole moment in an inhomogeneous electromagnetic field. In our context, the dipole moment arises from the spin of the particle. We show how to take the massless limit, obtaining a result that agrees with previous works $[15,16]$. We also study the solution of the Boltzmann equation in the case of global equilibrium with rigid rotation. Finally, we derive fluid-dynamical equations of motion with spin degrees of freedom from the Wigner function using the canonical definitions of the energymomentum and spin tensors. In accordance with previous works [36,40], the conservation of the total angular momentum is promoted as an additional fluid-dynamical equation, where the divergence of the spin tensor is related to the antisymmetric part of the energy-momentum tensor.

We use units $c=k_{B}=1$ throughout this paper. It is useful to explicitly keep Planck's constant $\hbar$, since it will be our power-counting parameter. The convention for the metric tensor is $g^{\mu \nu}=\operatorname{diag}(+1,-1,-1,-1)$ and $\epsilon^{0123}=$ $-\epsilon_{0123}=+1$ for the rank-four Levi-Civita tensor. We use the notation $a^{\mu} b_{\mu} \equiv a \cdot b$ for the scalar product of two fourvectors $a^{\mu}, b^{\mu}$ and $\mathbf{a} \cdot \mathbf{b}$ for the corresponding scalar product of two spatial vectors $\mathbf{a}, \mathbf{b}$. A two-dimensional vector in spin space is denoted by $\vec{a}$. The electromagnetic four-potential is $\mathbb{A}^{\mu}$, where the electromagnetic charge is absorbed into its definition. We denote the dipole-moment tensor as $\Sigma^{\mu \nu}$. This quantity corresponds to the spin tensor $S^{\mu \nu}$ of Refs. [11,13]. In this paper the term "spin tensor" is reserved for the rank-three Lorentz tensor $S^{\lambda, \mu \nu}$.

\section{EQUATIONS FOR THE WIGNER FUNCTION FOR MASSIVE FERMIONS}

The Wigner function is defined as the Fourier transform of the two-point correlation function [44],

$$
\begin{aligned}
W_{\alpha \beta}(x, p)= & \int \frac{d^{4} y}{(2 \pi \hbar)^{4}} e^{-\frac{i}{\hbar} p \cdot y} \\
& \times\left\langle: \bar{\psi}_{\beta}\left(x_{1}\right) U\left(x_{1}, x_{2}\right) \psi_{\alpha}\left(x_{2}\right):\right\rangle .
\end{aligned}
$$

Here, $x_{1}$ and $x_{2}$ are the space-time coordinates of two different points, with $y^{\mu} \equiv x_{1}^{\mu}-x_{2}^{\mu}$ and $x^{\mu} \equiv\left(x_{1}^{\mu}+x_{2}^{\mu}\right) / 2$. The gauge link is defined as

$$
U\left(x_{1}, x_{2}\right)=\exp \left[-\frac{i}{\hbar} y^{\mu} \int_{-1 / 2}^{1 / 2} d t \mathbb{A}_{\mu}(x+t y)\right] .
$$

In this paper, $\mathbb{A}_{\mu}$ will be treated as an external, classical field (otherwise, the gauge link would need to be pathordered). The particular choice of path for the integration between $x_{1}$ and $x_{2}$ ensures that $p^{\mu}$ is the kinetic momentum. Note that the factors $2 \pi \hbar$ in the denominator in Eq. (1) belong to the phase-space volume and do not participate in the $\hbar$-counting employed throughout this paper.

Starting from the Dirac equation and its adjoint,

$$
(i \hbar \gamma \cdot D-m) \psi=0=\bar{\psi}\left(i \hbar \gamma \cdot D^{\dagger}+m\right),
$$

where $D_{\mu} \equiv \partial_{x \mu}+\frac{i}{\hbar} \mathbb{A}_{\mu}$ is the covariant derivative, one can derive the kinetic equation for the Wigner function as [44]

$$
(\gamma \cdot K-m) W(x, p)=0 .
$$

Here one has defined the operator

$$
K^{\mu} \equiv \Pi^{\mu}+\frac{1}{2} i \hbar \nabla^{\mu}
$$

with the generalized space-time derivative and momentum operators

$$
\begin{aligned}
\nabla^{\mu} & \equiv \partial_{x}^{\mu}-j_{0}(\Delta) F^{\mu \nu} \partial_{p \nu}, \\
\Pi^{\mu} & \equiv p^{\mu}-\frac{\hbar}{2} j_{1}(\Delta) F^{\mu \nu} \partial_{p \nu},
\end{aligned}
$$

where $\Delta \equiv \frac{\hbar}{2} \partial_{p} \cdot \partial_{x}$ and $F^{\mu \nu}=\partial_{x}^{\mu} \mathbb{A}^{\nu}-\partial_{x}^{\nu} \mathbb{A}^{\mu}$ is the electromagnetic field-strength tensor. We should emphasize that in 
Eq. (4) the space-time derivative $\partial_{x}$ contained in $\Delta$ only acts on $F^{\mu \nu}$, but not on the Wigner function. The functions $j_{0}(x)=\sin x / x$ and $j_{1}(x)=(\sin x-x \cos x) / x^{2}$ are spherical Bessel functions. If we assume that the particles only interact with the classical electromagnetic field but not among themselves (which, in the language of kinetic theory, is the limit of the collisionless Boltzmann-Vlasov equation), Eq. (4) is exact and contains the full dynamics of the Wigner function.

In order to derive a kinetic equation for massive spin-1/2 particles, it is advantageous to decompose the Wigner function in terms of a basis formed by the 16 independent generators of the Clifford algebra $\left\{1, \gamma^{5}, \gamma^{\mu}, \gamma^{5} \gamma^{\mu}, \sigma^{\mu \nu}\right\}$, with $\gamma^{5} \equiv i \gamma^{0} \gamma^{1} \gamma^{2} \gamma^{3}$ and $\sigma^{\mu \nu} \equiv \frac{i}{2}\left[\gamma^{\mu}, \gamma^{\nu}\right]$,

$W=\frac{1}{4}\left(\mathcal{F}+i \gamma^{5} \mathcal{P}+\gamma^{\mu} \mathcal{V}_{\mu}+\gamma^{5} \gamma^{\mu} \mathcal{A}_{\mu}+\frac{1}{2} \sigma^{\mu \nu} \mathcal{S}_{\mu \nu}\right)$.

The coefficients $\mathcal{F}, \mathcal{P}, \mathcal{V}^{\mu}, \mathcal{A}^{\mu}$, and $\mathcal{S}^{\mu \nu}$ are real functions of the phase-space coordinates $x, p$ and correspond to the scalar, pseudoscalar, vector, axial-vector, and tensor components of the Wigner function. Some of them have an obvious physical meaning [52]. For example, $\mathcal{V}^{\mu}$ is the fermion four-current and $\mathcal{A}^{\mu}$ is related to the spin density. Using the trace properties of the Dirac matrices, the coefficients in Eq. (8) are given by

$$
\begin{aligned}
\mathcal{F} & =\operatorname{Tr}(W), \\
\mathcal{P} & =-i \operatorname{Tr}\left(\gamma^{5} W\right), \\
\mathcal{V}^{\mu} & =\operatorname{Tr}\left(\gamma^{\mu} W\right), \\
\mathcal{A}^{\mu} & =\operatorname{Tr}\left(\gamma^{\mu} \gamma^{5} W\right), \\
\mathcal{S}^{\mu \nu} & =\operatorname{Tr}\left(\sigma^{\mu \nu} W\right) .
\end{aligned}
$$

Replacing $W$ in Eq. (4) by the decomposition (8), we find the following complex-valued equations:

$$
\begin{aligned}
K \cdot \mathcal{V}-m \mathcal{F} & =0, \\
K \cdot \mathcal{A}+i m \mathcal{P} & =0, \\
K_{\mu} \mathcal{F}+i K^{\nu} \mathcal{S}_{\nu \mu}-m \mathcal{V}_{\mu} & =0, \\
i K_{\mu} \mathcal{P}+\frac{1}{2} \epsilon_{\mu \nu \alpha \beta} K^{\nu} \mathcal{S}^{\alpha \beta}+m \mathcal{A}_{\mu} & =0, \\
-i K_{[\mu} \mathcal{V}_{\nu]}-\epsilon_{\mu \nu \alpha \beta} K^{\alpha} \mathcal{A}^{\beta}-m \mathcal{S}_{\mu \nu} & =0,
\end{aligned}
$$

where $A_{[\mu} B_{\nu]} \equiv A_{\mu} B_{\nu}-A_{\nu} B_{\mu}$. Decomposing these equations into their real and imaginary parts, we obtain a set of coupled equations which determine the coefficients in the decomposition (8) of the Wigner function. The real parts read

$$
\begin{aligned}
\Pi \cdot \mathcal{V}-m \mathcal{F} & =0, \\
\frac{\hbar}{2} \nabla \cdot \mathcal{A}+m \mathcal{P} & =0,
\end{aligned}
$$

$$
\begin{gathered}
\Pi_{\mu} \mathcal{F}-\frac{\hbar}{2} \nabla^{\nu} \mathcal{S}_{\nu \mu}-m \mathcal{V}_{\mu}=0 \\
-\frac{\hbar}{2} \nabla_{\mu} \mathcal{P}+\frac{1}{2} \epsilon_{\mu \nu \alpha \beta} \Pi^{\nu} S^{\alpha \beta}+m \mathcal{A}_{\mu}=0 \\
\frac{\hbar}{2} \nabla_{[\mu} \mathcal{V}_{\nu]}-\epsilon_{\mu \nu \alpha \beta} \Pi^{\alpha} \mathcal{A}^{\beta}-m \mathcal{S}_{\mu \nu}=0
\end{gathered}
$$

and the imaginary parts are

$$
\begin{gathered}
\hbar \nabla \cdot \mathcal{V}=0, \\
\Pi \cdot \mathcal{A}=0, \\
\frac{\hbar}{2} \nabla_{\mu} \mathcal{F}+\Pi^{\nu} \mathcal{S}_{\nu \mu}=0, \\
\Pi_{\mu} \mathcal{P}+\frac{\hbar}{4} \epsilon_{\mu \nu \alpha \beta} \nabla^{\nu} \mathcal{S}^{\alpha \beta}=0, \\
\prod_{[\mu} \mathcal{V}_{\nu]}+\frac{\hbar}{2} \epsilon_{\mu \nu \alpha \beta} \nabla^{\alpha} \mathcal{A}^{\beta}=0 .
\end{gathered}
$$

In the next sections, we will explicitly solve Eqs. (11)-(20) to zeroth order in $\hbar$, and then derive kinetic equations which the general solution has to fulfill up to first order in $\hbar$.

\section{ZEROTH-ORDER SOLUTION}

To zeroth order in $\hbar$, the operator $K^{\mu}=p^{\mu}$ and Eq. (4) reduces to

$$
(\gamma \cdot p-m) W^{(0)}(x, p)=0 .
$$

The solution is given by $[41,50]$

$$
W_{\alpha \beta}^{(0)}(x, p)=W_{\alpha \beta}^{+}(x, p)+W_{\alpha \beta}^{-}(x, p),
$$

where

$$
\begin{aligned}
W_{\alpha \beta}^{+}(x, p)= & \frac{1}{(2 \pi \hbar)^{3}} \int d^{4} q \theta\left(q^{0}\right) \delta\left(q^{2}-m^{2}\right) \delta^{4}(p-q) \\
& \times \sum_{r s} u_{\alpha}(\mathbf{q}, r) \bar{u}_{\beta}(\mathbf{q}, s) f_{r s}^{(0)+}(x, \mathbf{q}) \\
W_{\alpha \beta}^{-}(x, p)= & -\frac{1}{(2 \pi \hbar)^{3}} \int d^{4} q \theta\left(q^{0}\right) \delta\left(q^{2}-m^{2}\right) \delta^{4}(p+q) \\
& \times \sum_{r s} v_{\alpha}(\mathbf{q}, s) \bar{v}_{\beta}(\mathbf{q}, r) f_{s r}^{(0)-}(x, \mathbf{q})
\end{aligned}
$$

are the contributions from positive and negative energies, respectively. Here, $f_{r s}^{+}(x, \mathbf{q})$ and $f_{s r}^{-}(x, \mathbf{q})$ are the distribution functions for fermions and antifermions, respectively, which are in general matrices in spin space. The spin indices label spin states parallel, $r, s=+$, or antiparallel, 
$r, s=-$, to the quantization direction in the rest frame of the particle, respectively.

This spin quantization direction can in principle be chosen arbitrarily. However, the most convenient choice is to quantize the spin with respect to the polarization direction $[41,45]$. In other words, we choose a spin basis in which the new distribution functions $\tilde{f}_{r s}^{ \pm}$are diagonal, i.e.,

$$
\tilde{f}_{r s}^{(0) \pm}=f_{s}^{(0) \pm} \delta_{r s} .
$$

In Appendix A we demonstrate that such a choice is always possible, at the expense of introducing space-time dependent spinors, cf. Eq. (A7). We will also use the diagonal basis in the calculation of the contributions of higher order in $\hbar$ in the following sections.

As shown in Appendix A, the spin quantization direction $n^{(0) \mu}$ is given by

$$
n^{(0) \mu}(x, p) \equiv \theta\left(p^{0}\right) n^{+\mu}(x, \mathbf{p})-\theta\left(-p^{0}\right) n^{-\mu}(x, \mathbf{p}),
$$

where

$$
\begin{aligned}
& n^{+\mu}(x, \mathbf{p})=\left(\frac{\mathbf{n}^{+} \cdot \mathbf{p}}{m}, \mathbf{n}^{+}+\frac{\mathbf{n}^{+} \cdot \mathbf{p}}{m\left(m+E_{\mathbf{p}}\right)} \mathbf{p}\right) \\
& n^{-\mu}(x, \mathbf{p})=\left(\frac{\mathbf{n}^{-} \cdot \mathbf{p}}{m},-\mathbf{n}^{-}-\frac{\mathbf{n}^{-} \cdot \mathbf{p}}{m\left(m+E_{\mathbf{p}}\right)} \mathbf{p}\right) .
\end{aligned}
$$

Here, $\mathbf{n}^{ \pm}$is the spin quantization direction in the rest frame of the particle/antiparticle [cf. Eq. (A6)] and $E_{\mathbf{p}}=$ $\sqrt{\mathbf{p}^{2}+m^{2}}$. The spin quantization direction $\mathbf{n}^{ \pm}$transforms as an axial vector under Lorentz boosts and parity transformations. We show in Appendix A that $\mathbf{n}^{ \pm}$depends in general on $\mathbf{p}$ and $x$, thus $n^{ \pm \mu}$ is defined locally. The vector $n^{(0) \mu}$ is aligned with the polarization direction and agrees with the classical spin vector, i.e., as we will see later, it obeys the classical equation for spin precession in an electromagnetic field, the so-called Bargmann-Michel-Telegdi (BMT) equation [53]. Moreover, $n^{(0) \mu}$ fulfills $p \cdot n^{(0)}=0$ (which can be seen using Eqs. (A9) and (A10) and applying the Dirac equation for the $u$-and $v$-spinors as well as the identity $\left.\bar{u}(\mathbf{p}, r) \gamma^{5} u(\mathbf{p}, s)=\bar{v}(-\mathbf{p}, r) \gamma^{5} v(-\mathbf{p}, s)=0\right)$.

Equations (22)-(24) represent the solution obtained in Ref. [50] for vanishing electromagnetic fields. However, this is also the solution for nonvanishing electromagnetic fields, since the form of Eq. (4) remains the same. The momentum variable $p^{\mu}$ is then the kinetic (and not the canonical) momentum.

Closer inspection of Eq. (4) reveals that Eq. (22) with Eqs. (23), (24) is also a solution to Eq. (4) at arbitrary order in $\hbar$, if $\gamma \cdot \nabla W^{(0)}=0$ and $\gamma_{\mu} F^{\mu \nu} \partial_{p \nu} W^{(0)}=0$ (because then the $\hbar$-dependence of the operator $K^{\mu}$ vanishes). In the absence of electromagnetic fields, one at least needs to require that $\gamma \cdot \partial_{x} W^{(0)}=0$. In the full solution, i.e., the solution to all orders in $\hbar$, the momentum variable $q$ is no longer equal to the kinetic momentum $p$. This is obviously not the case for Eqs. (23) and (24), since they are proportional to $\sim \delta^{4}(p \mp q)$, see also the discussion in Ref. [50].

Now we easily obtain the coefficients of the decomposition (8) using Eqs. (9) and (25). We find

$\mathcal{F}^{(0)}(x, p)=m \delta\left(p^{2}-m^{2}\right) V^{(0)}(x, p)$,

$\mathcal{P}^{(0)}(x, p)=0$,

$\mathcal{V}_{\mu}^{(0)}(x, p)=p_{\mu} \delta\left(p^{2}-m^{2}\right) V^{(0)}(x, p)$,

$\mathcal{A}_{\mu}^{(0)}(x, p)=m n_{\mu}^{(0)}(x, p) \delta\left(p^{2}-m^{2}\right) A^{(0)}(x, p)$,

$\mathcal{S}_{\mu \nu}^{(0)}(x, p)=m \Sigma_{\mu \nu}^{(0)}(x, p) \delta\left(p^{2}-m^{2}\right) A^{(0)}(x, p)$,

with

$$
V^{(0)}(x, p) \equiv \frac{2}{(2 \pi \hbar)^{3}} \sum_{e s} \theta\left(e p^{0}\right) f_{s}^{(0) e}(x, e \mathbf{p})
$$

and

$$
A^{(0)}(x, p) \equiv \frac{2}{(2 \pi \hbar)^{3}} \sum_{e s} s \theta\left(e p^{0}\right) f_{s}^{(0) e}(x, e \mathbf{p})
$$

where $e= \pm, f_{s}^{(0) e}$ are the distribution functions in the diagonal basis, and the dipole-moment tensor is defined as

$$
\Sigma_{\mu \nu}^{(0)}(x, p)=-\frac{1}{m} \epsilon_{\mu \nu \alpha \beta} p^{\alpha} n^{(0) \beta},
$$

for the proof, see Appendix A.

\section{GENERAL SOLUTION UP TO ORDER $\hbar$}

In this section we derive the general solution for Eqs. (11)-(20) to first order in $\hbar$. We emphasize that these equations are not independent from each other. We prove in Appendix B that Eq. (18) can be derived from Eqs. (11), (15), (16), (20), and (19) can be derived from Eqs. (12), (15), (17), and(20). Thus, one can ignore Eqs. (18) and (19) when solving this system of partial differential equations.

Using Eqs. (12), (13) and, (14) one can express the pseudoscalar, vector, and axial-vector parts $\mathcal{P}, \mathcal{V}^{\mu}$, and $\mathcal{A}^{\mu}$ as follows:

$$
\begin{aligned}
\mathcal{P} & =-\frac{\hbar}{2 m} \nabla \cdot \mathcal{A}, \\
\mathcal{V}_{\mu} & =\frac{1}{m} \Pi_{\mu} \mathcal{F}-\frac{\hbar}{2 m} \nabla^{\nu} \mathcal{S}_{\nu \mu}, \\
\mathcal{A}_{\mu} & =\frac{\hbar}{2 m} \nabla_{\mu} \mathcal{P}-\frac{1}{2 m} \epsilon_{\mu \nu \alpha \beta} \Pi^{\nu} \mathcal{S}^{\alpha \beta} .
\end{aligned}
$$

Inserting them back into Eqs. (11) and (15) one obtains the modified on-shell conditions for the scalar and tensor components, 


$$
\begin{aligned}
\left(\Pi \cdot \Pi-m^{2}\right) \mathcal{F}= & \frac{\hbar}{2} \Pi^{\mu} \nabla^{\nu} \mathcal{S}_{\nu \mu}, \\
\left(\Pi \cdot \Pi-m^{2}\right) \mathcal{S}_{\mu \nu}= & -\Pi^{\alpha} \Pi_{[\mu} \mathcal{S}_{\nu] \alpha}-\frac{\hbar}{2} \nabla_{[\mu} \Pi_{\nu]} \mathcal{F}-\frac{\hbar^{2}}{4} \nabla_{[\mu} \nabla^{\alpha} \mathcal{S}_{\nu] \alpha} \\
& +\frac{\hbar}{2} \epsilon_{\mu \nu \alpha \beta} \Pi^{\alpha} \nabla^{\beta} \mathcal{P} .
\end{aligned}
$$

Equations (32) and (33) are equivalent to Eqs. (11)-(15). In general, the right-hand sides are nonvanishing, which indicates that the Wigner function contains offshell effects.

From their definitions (6), (7), we observe that the operators $\nabla^{\mu}$ and $\Pi^{\mu}$ can be expanded in a series of powers in $\hbar^{2}$. In order to derive the semiclassical limit, we may truncate these series at order $\hbar^{0}$ and $\hbar^{2}$, respectively,

$$
\begin{aligned}
& \nabla^{\mu}=\sum_{n=0}^{\infty} \hbar^{2 n} \nabla^{(2 n) \mu}=\nabla^{(0) \mu}+\mathcal{O}\left(\hbar^{2}\right), \\
& \Pi^{\mu}=\sum_{n=0}^{\infty} \hbar^{2 n} \Pi^{(2 n) \mu}=p^{\mu}-\frac{\hbar^{2}}{12}\left(\partial_{x}^{\alpha} F^{\mu \nu}\right) \partial_{p \nu} \partial_{p \alpha}+\mathcal{O}\left(\hbar^{4}\right),
\end{aligned}
$$

where $\nabla^{(0) \mu} \equiv \partial_{x}^{\mu}-F^{\mu \nu} \partial_{p \nu}$. We also expand the functions $\mathcal{F}, \mathcal{P}, \mathcal{V}^{\mu}, \mathcal{A}^{\mu}, \mathcal{S}^{\mu \nu}$ into power series in $\hbar$, e.g.,

$$
\mathcal{F}=\sum_{n=0}^{\infty} \hbar^{n} \mathcal{F}^{(n)}
$$

Inserting these expansions into Eqs. (11)-(20) and then comparing order by order in $\hbar$ one can get a set of equations which we will analyze up to second order in $\hbar$ in the remainder of this section.

\section{A. Zeroth order in $\hbar$}

We first analyze the on-shell conditions (33) for the scalar and tensor components to leading order in $\hbar$ and show that the direct calculation of the Wigner function to this order presented in Sec. III is consistent with these conditions. To order $\mathcal{O}\left(\hbar^{0}\right)$, Eq. (33) reads

$$
\begin{aligned}
\left(p^{2}-m^{2}\right) \mathcal{F}^{(0)} & =0, \\
\left(p^{2}-m^{2}\right) \mathcal{S}_{\mu \nu}^{(0)} & =0,
\end{aligned}
$$

where we have used $p^{\nu} \mathcal{S}_{\nu \mu}^{(0)}=0$, which is the constraint equation (18) to zeroth order in $\hbar$. The general solution of the above equations reads

$$
\begin{aligned}
\mathcal{F}^{(0)} & =m V^{(0)} \delta\left(p^{2}-m^{2}\right), \\
\mathcal{S}_{\mu \nu}^{(0)} & =m \Sigma_{\mu \nu}^{(0)} A^{(0)} \delta\left(p^{2}-m^{2}\right),
\end{aligned}
$$

where $V^{(0)}, \Sigma_{\mu \nu}^{(0)} A^{(0)}$ are up to now arbitrary functions which do not have singularities at $p^{2}=m^{2}$. We also demand that they go to zero sufficiently fast for large momenta (in order to neglect boundary terms when performing an integration by parts). Comparing to the previous section, we can identify $V^{(0)}$ with the spin-symmetric combination (29) and $A^{(0)}$ with spin-antisymmetric combination (30) of the zeroth-order distribution function, as well as $\Sigma_{\mu \nu}^{(0)}$ with the dipole-moment tensor, which satisfies $p^{\mu} \Sigma_{\mu \nu}^{(0)}=0$ in order to fulfill Eq. (18). In order to be consistent with Eq. (31), we demand $\Sigma^{(0) \mu \nu} \Sigma_{\mu \nu}^{(0)}=2$.

With the help of Eq. (32) we can now write down the remaining components of the Wigner function to leading order in $\hbar$,

$$
\begin{aligned}
& \mathcal{P}^{(0)}=0, \\
& \mathcal{V}_{\mu}^{(0)}=p_{\mu} V^{(0)} \delta\left(p^{2}-m^{2}\right), \\
& \mathcal{A}_{\mu}^{(0)}=-\frac{1}{2} \epsilon_{\mu \nu \alpha \beta} p^{\nu} \Sigma^{(0) \alpha \beta} A^{(0)} \delta\left(p^{2}-m^{2}\right) .
\end{aligned}
$$

It is straightforward to check that our solutions (37), (38) satisfy Eqs. (16)-(20). All zeroth-order solutions are on mass-shell and agree with the results from the direct calculation of the Wigner function in Sec. III.

\section{B. First order in $\hbar$}

The starting point for our analysis of the contributions of next-to-leading order in $\hbar$ is again the on-shell equation (33). The $\mathcal{O}(\hbar)$ part reads

$$
\begin{aligned}
\left(p^{2}-m^{2}\right) \mathcal{F}^{(1)} & =\frac{1}{2} p^{\mu} \nabla^{(0) \nu} \mathcal{S}_{\nu \mu}^{(0)}=\frac{1}{2} F^{\mu \nu} \mathcal{S}_{\mu \nu}^{(0)}, \\
\left(p^{2}-m^{2}\right) \mathcal{S}_{\mu \nu}^{(1)} & =-p^{\alpha} p_{[\mu} \mathcal{S}_{\nu] \alpha}^{(1)}-\frac{1}{2} \nabla_{[\mu}^{(0)} p_{\nu]} \mathcal{F}^{(0)}=F_{\mu \nu} \mathcal{F}^{(0)},
\end{aligned}
$$

where we used $p^{\mu} \mathcal{S}_{\nu \mu}^{(0)}=0$ and the relation

$$
p^{\nu} \mathcal{S}_{\mu \nu}^{(1)}=\frac{1}{2} \nabla_{\mu}^{(0)} \mathcal{F}^{(0)},
$$

which follows from Eq. (18) to first order in $\hbar$. Here the leading-order functions $\mathcal{S}_{\mu \nu}^{(0)}$ and $\mathcal{F}^{(0)}$ have been obtained in the previous subsection. The solutions to Eq. (39) can in general be written as

$$
\begin{aligned}
& \mathcal{F}^{(1)}=m\left[V^{(1)} \delta\left(p^{2}-m^{2}\right)-\frac{1}{2} F^{\mu \nu} \Sigma_{\mu \nu}^{(0)} A^{(0)} \delta^{\prime}\left(p^{2}-m^{2}\right)\right], \\
& \mathcal{S}_{\mu \nu}^{(1)}=m\left[\bar{\Sigma}_{\mu \nu}^{(1)} \delta\left(p^{2}-m^{2}\right)-F_{\mu \nu} V^{(0)} \delta^{\prime}\left(p^{2}-m^{2}\right)\right] .
\end{aligned}
$$

Here, $\bar{\Sigma}_{\mu \nu}^{(1)}$ is, up to a factor $m$, the on-shell part of the firstorder dipole moment. We note that $\bar{\Sigma}_{\mu \nu}^{(1)}$ is not normalized. 
The functions $V^{(1)}$ and $\bar{\Sigma}_{\mu \nu}^{(1)}$ will be determined from the kinetic equations that we will derive below. The function $V^{(1)}$ can be identified as the $\mathcal{O}(\hbar)$ correction to the spinsymmetric combination of the distribution function. Using Eq. (40), we derive a constraint for $\bar{\Sigma}_{\mu \nu}^{(1)}$,

$$
p^{\nu} \bar{\Sigma}_{\mu \nu}^{(1)} \delta\left(p^{2}-m^{2}\right)=\frac{1}{2} \delta\left(p^{2}-m^{2}\right) \nabla_{\mu}^{(0)} V^{(0)}
$$

Expanding all quantities in Eq. (32) into power series in $\hbar$, to $\mathcal{O}(\hbar)$ we obtain

$$
\begin{aligned}
\mathcal{P}^{(1)} & =-\frac{1}{2 m} \nabla^{(0) \mu} \mathcal{A}_{\mu}^{(0)}, \\
\mathcal{V}_{\mu}^{(1)} & =\frac{p_{\mu}}{m} \mathcal{F}^{(1)}-\frac{1}{2 m} \nabla^{(0) \nu} \mathcal{S}_{\nu \mu}^{(0)}, \\
\mathcal{A}_{\mu}^{(1)} & =\frac{1}{2 m} \nabla_{\mu}^{(0)} \mathcal{P}^{(0)}-\frac{1}{2 m} \epsilon_{\mu \nu \alpha \beta} p^{\nu} \mathcal{S}^{(1) \alpha \beta} .
\end{aligned}
$$

Inserting the zeroth- and first-order solutions from Eqs. (37), (38), and (41), we can derive the first-order pseudoscalar, vector, and axial-vector functions,

$$
\begin{aligned}
\mathcal{P}^{(1)}= & \frac{1}{4 m} \epsilon^{\mu \nu \alpha \beta} \nabla_{\mu}^{(0)}\left[p_{\nu} \Sigma_{\alpha \beta}^{(0)} A^{(0)} \delta\left(p^{2}-m^{2}\right)\right], \\
\mathcal{V}_{\mu}^{(1)}= & \delta\left(p^{2}-m^{2}\right)\left[p_{\mu} V^{(1)}+\frac{1}{2} \nabla^{(0) \nu} \Sigma_{\mu \nu}^{(0)} A^{(0)}\right] \\
& -\left[\frac{1}{2} p_{\mu} F^{\alpha \beta} \Sigma_{\alpha \beta}^{(0)}+\Sigma_{\mu \nu}^{(0)} F^{\nu \alpha} p_{\alpha}\right] A^{(0)} \delta^{\prime}\left(p^{2}-m^{2}\right), \\
\mathcal{A}_{\mu}^{(1)}= & m \bar{n}_{\mu}^{(1)} \delta\left(p^{2}-m^{2}\right)+\tilde{F}_{\mu \nu} p^{\nu} V^{(0)} \delta^{\prime}\left(p^{2}-m^{2}\right),
\end{aligned}
$$

where

$$
\bar{n}_{\mu}^{(1)} \equiv-\frac{1}{2 m} \epsilon_{\mu \nu \alpha \beta} p^{\nu} \bar{\Sigma}^{(1) \alpha \beta}
$$

is the first-order on-shell correction to $n_{\mu}^{(0)} A^{(0)}$.

To first order in $\hbar$, the constraints (16), (20) read

$$
\begin{aligned}
\nabla^{(0)} \cdot \mathcal{V}^{(0)} & =0, \\
p_{[\mu} \mathcal{V}_{\nu]}^{(1)}+\frac{1}{2} \epsilon_{\mu \nu \alpha \beta} \nabla^{(0) \alpha} \mathcal{A}^{(0) \beta} & =0 .
\end{aligned}
$$

They lead to the kinetic equations of the particle distributions and the dipole moment to zeroth order in $\hbar$; for details see Appendix C,

$$
\begin{aligned}
\delta\left(p^{2}-m^{2}\right) p \cdot \nabla^{(0)} V^{(0)} & =0, \\
\delta\left(p^{2}-m^{2}\right) p \cdot \nabla^{(0)} A^{(0)} & =0, \\
\delta\left(p^{2}-m^{2}\right)\left[p \cdot \nabla^{(0)} \Sigma_{\mu \nu}^{(0)}-F_{[\mu}^{\alpha} \Sigma_{\nu] \alpha}^{(0)}\right] & =0 .
\end{aligned}
$$

\section{Second order in $\hbar$}

As we have shown in the previous subsection, the zerothorder kinetic equations are derived from the first-order constraint equations. In order to obtain the first-order kinetic equations, we focus on the second-order parts of Eqs. (16) and (20),

$$
\begin{aligned}
\nabla^{(0)} \cdot \mathcal{V}^{(1)} & =0, \\
p_{[\mu} \mathcal{V}_{\nu]}^{(2)}+\Pi_{[\mu}^{(2)} \mathcal{V}_{\nu]}^{(0)}+\frac{1}{2} \epsilon_{\mu \nu \alpha \beta} \nabla^{(0) \alpha} \mathcal{A}^{(1) \beta} & =0,
\end{aligned}
$$

with the operator $\Pi_{\mu}^{(2)}=-\frac{1}{12}\left(\partial_{x \alpha} F_{\mu \nu}\right) \partial_{p}^{\alpha} \partial_{p}^{\nu}$. After some calculation (cf. Appendix C), one derives the following kinetic equations,

$$
\begin{aligned}
0= & \delta\left(p^{2}-m^{2}\right)\left[p \cdot \nabla^{(0)} V^{(1)}+\frac{1}{4}\left(\partial_{x}^{\alpha} F^{\mu \nu}\right) \partial_{p \alpha}\left(\Sigma_{\mu \nu}^{(0)} A^{(0)}\right)\right] \\
- & \frac{1}{2} \delta^{\prime}\left(p^{2}-m^{2}\right) F^{\alpha \beta} p \cdot \nabla^{(0)}\left(\Sigma_{\alpha \beta}^{(0)} A^{(0)}\right), \\
0= & \delta\left(p^{2}-m^{2}\right)\left[p \cdot \nabla^{(0)} \bar{\Sigma}_{\mu \nu}^{(1)}-F_{[\mu}^{\alpha} \bar{\Sigma}_{\nu] \alpha}^{(1)}+\frac{1}{2}\left(\partial_{x \alpha} F_{\mu \nu}\right) \partial_{p}^{\alpha} V^{(0)}\right] \\
& -\delta^{\prime}\left(p^{2}-m^{2}\right) F_{\mu \nu} p \cdot \nabla^{(0)} V^{(0)} .
\end{aligned}
$$

Multiplying the second equation (49) by $-\frac{1}{2 m} \epsilon^{\alpha \beta \mu \nu} p_{\beta}$ and using Eq. (45), we obtain a kinetic equation for $\bar{n}_{\mu}^{(1)}$,

$$
\begin{aligned}
0= & \delta\left(p^{2}-m^{2}\right)\left[p \cdot \nabla^{(0)} \bar{n}_{\mu}^{(1)}-F_{\mu \nu} \bar{n}^{(1) \nu}\right. \\
& \left.-\frac{1}{2 m} p^{\nu}\left(\partial_{x \alpha} \tilde{F}_{\mu \nu}\right) \partial_{p}^{\alpha} V^{(0)}\right] \\
& +\delta^{\prime}\left(p^{2}-m^{2}\right) \frac{1}{m} \tilde{F}_{\mu \nu} p^{\nu} p \cdot \nabla^{(0)} V^{(0)},
\end{aligned}
$$

where $\tilde{F}_{\mu \nu} \equiv \frac{1}{2} \epsilon_{\mu \nu \alpha \beta} F^{\alpha \beta}$ is the dual field-strength tensor.

\section{KINETIC EQUATIONS FOR spin-1/2 PARTICLES}

In order to summarize our results in a compact form, we define the resummed functions

$$
\begin{aligned}
V & \equiv V^{(0)}+\hbar V^{(1)}+\mathcal{O}\left(\hbar^{2}\right), \\
\bar{\Sigma}^{\mu \nu} & \equiv \Sigma^{(0) \mu \nu} A^{(0)}+\hbar \bar{\Sigma}^{(1) \mu \nu}+\mathcal{O}\left(\hbar^{2}\right) .
\end{aligned}
$$

Using these resummed functions, the components the Wigner function, given by Eqs. (37) and (38) to zeroth order in $\hbar$ and by Eqs. (41) and (44) to first order in $\hbar$, can be written as 


$$
\begin{aligned}
\mathcal{F}= & m\left[V \delta\left(p^{2}-m^{2}\right)-\frac{\hbar}{2} F^{\mu \nu} \bar{\Sigma}_{\mu \nu} \delta^{\prime}\left(p^{2}-m^{2}\right)\right]+\mathcal{O}\left(\hbar^{2}\right), \\
\mathcal{P}= & \frac{\hbar}{4 m} \epsilon^{\mu \nu \alpha \beta} \nabla_{\mu}^{(0)}\left[p_{\nu} \bar{\Sigma}_{\alpha \beta} \delta\left(p^{2}-m^{2}\right)\right]+\mathcal{O}\left(\hbar^{2}\right), \\
\mathcal{V}_{\mu}= & p_{\mu}\left[V \delta\left(p^{2}-m^{2}\right)-\frac{\hbar}{2} F^{\alpha \beta} \bar{\Sigma}_{\alpha \beta} \delta^{\prime}\left(p^{2}-m^{2}\right)\right] \\
& +\frac{\hbar}{2} \nabla^{(0) \nu}\left[\bar{\Sigma}_{\mu \nu} \delta\left(p^{2}-m^{2}\right)\right]+\mathcal{O}\left(\hbar^{2}\right), \\
\mathcal{A}_{\mu}= & -\frac{1}{2} \epsilon_{\mu \nu \alpha \beta} p^{\nu}\left[\bar{\Sigma}^{\alpha \beta} \delta\left(p^{2}-m^{2}\right)-\hbar F^{\alpha \beta} V \delta^{\prime}\left(p^{2}-m^{2}\right)\right] \\
& +\mathcal{O}\left(\hbar^{2}\right), \\
\mathcal{S}_{\mu \nu}= & m\left[\bar{\Sigma}_{\mu \nu} \delta\left(p^{2}-m^{2}\right)-\hbar F_{\mu \nu} V \delta^{\prime}\left(p^{2}-m^{2}\right)\right]+\mathcal{O}\left(\hbar^{2}\right) .
\end{aligned}
$$

The undetermined functions $V$ and $\bar{\Sigma}_{\mu \nu}$ satisfy one constraint equation,

$p^{\nu} \bar{\Sigma}_{\mu \nu} \delta\left(p^{2}-m^{2}\right)=\frac{\hbar}{2} \delta\left(p^{2}-m^{2}\right) \nabla_{\mu}^{(0)} V+\mathcal{O}\left(\hbar^{2}\right)$,

and two kinetic equations, which are the sum of Eqs. (47) and (49),

$$
\begin{aligned}
0= & \delta\left(p^{2}-m^{2}\right)\left[p \cdot \nabla^{(0)} V+\frac{\hbar}{4}\left(\partial_{x}^{\alpha} F^{\mu \nu}\right) \partial_{p \alpha} \bar{\Sigma}_{\mu \nu}\right] \\
& -\frac{\hbar}{2} \delta^{\prime}\left(p^{2}-m^{2}\right) F^{\alpha \beta} p \cdot \nabla^{(0)} \bar{\Sigma}_{\alpha \beta}+\mathcal{O}\left(\hbar^{2}\right), \\
0= & \delta\left(p^{2}-m^{2}\right)\left[p \cdot \nabla^{(0)} \bar{\Sigma}_{\mu \nu}-F_{[\mu}^{\alpha} \bar{\Sigma}_{\nu] \alpha}+\frac{\hbar}{2}\left(\partial_{x \alpha} F_{\mu \nu}\right) \partial_{p}^{\alpha} V\right] \\
& -\hbar \delta^{\prime}\left(p^{2}-m^{2}\right) F_{\mu \nu} p \cdot \nabla^{(0)} V+\mathcal{O}\left(\hbar^{2}\right) .
\end{aligned}
$$

Up to first order, we find that Eqs. (52), (53), and (54) are invariant under the following transformation

$$
\begin{aligned}
\bar{\Sigma}_{\mu \nu} & \rightarrow \hat{\bar{\Sigma}}_{\mu \nu}=\bar{\Sigma}_{\mu \nu}+\left(p^{2}-m^{2}\right) \delta \bar{\Sigma}_{\mu \nu}, \\
V & \rightarrow \hat{V}=V-\frac{\hbar}{2} F^{\mu \nu} \delta \bar{\Sigma}_{\mu \nu},
\end{aligned}
$$

or the transformation

$$
\begin{aligned}
V & \rightarrow \hat{V}=V+\left(p^{2}-m^{2}\right) \delta V, \\
\bar{\Sigma}_{\mu \nu} & \rightarrow \hat{\bar{\Sigma}}_{\mu \nu}=\bar{\Sigma}_{\mu \nu}-\hbar F_{\mu \nu} \delta V .
\end{aligned}
$$

Here $\delta \bar{\Sigma}_{\mu \nu}$ and $\delta V$ are arbitrary functions, which should be nonsingular on the mass-shell $p^{2}=m^{2}$. The invariance can be easily proved by using the property of the Dirac $\delta$-function $-x \delta^{\prime}(x)=\delta(x)$. Note that the first (second) transformation does not affect the on-shell value of $\bar{\Sigma}_{\mu \nu}(V)$ because the factor $p^{2}-m^{2}$ in front of $\delta \bar{\Sigma}_{\mu \nu}(\delta V)$ vanishes on the mass-shell.
It is possible to show that without loss of generality one can omit the terms proportional to the derivative of the delta function in the kinetic equations (54). In order to prove this, let us consider the $p^{0}$-integrated version of the last term in the second kinetic equation (54). For any function $G(x, p)$, we have

$$
\begin{aligned}
& \int d p^{0} \delta^{\prime}\left(p^{2}-m^{2}\right) G(x, p) p \cdot \nabla^{(0)} V \\
& =\int d p^{0} \frac{1}{2 p^{0}} G(x, p)\left[\partial_{p^{0}} \delta\left(p^{2}-m^{2}\right)\right] p \cdot \nabla^{(0)} V \\
& =-\int d p^{0} \frac{1}{2 p^{0}} \delta\left(p^{2}-m^{2}\right) G(x, p) \partial_{p^{0}} p \cdot \nabla^{(0)} V+\mathcal{O}(\hbar),
\end{aligned}
$$

where we integrated by parts in the last step and used Eq. (47). Applying the transformation (56) to Eq. (57) and choosing $\delta V$ such that

$\delta\left(p^{2}-m^{2}\right) 2 p^{0} p \cdot \nabla^{(0)} \delta V=-\delta\left(p^{2}-m^{2}\right) \partial_{p^{0}} p \cdot \nabla^{(0)} V$,

(where we assume that $\delta V$ is nonsingular at $p^{2}=m^{2}$ ) we find

$$
\int d p^{0} \delta^{\prime}\left(p^{2}-m^{2}\right) G(x, p) p \cdot \nabla^{(0)} \hat{V}=\mathcal{O}(\hbar) .
$$

A similar procedure can be applied to the first kinetic equation (54). This proves that the terms proportional to the derivative of the delta function in the kinetic equations (54) are actually of order $O\left(\hbar^{2}\right)$, and we obtain

$$
\begin{aligned}
0= & \delta\left(p^{2}-m^{2}\right)\left[p \cdot \nabla^{(0)} \hat{V}+\frac{\hbar}{4}\left(\partial_{x}^{\alpha} F^{\mu \nu}\right) \partial_{p \alpha} \hat{\bar{\Sigma}}_{\mu \nu}\right]+\mathcal{O}\left(\hbar^{2}\right), \\
0= & \delta\left(p^{2}-m^{2}\right)\left[p \cdot \nabla^{(0)} \hat{\bar{\Sigma}}_{\mu \nu}-F_{[\mu}^{\alpha} \hat{\bar{\Sigma}}_{\nu] \alpha}+\frac{\hbar}{2}\left(\partial_{x \alpha} F_{\mu \nu}\right) \partial_{p}^{\alpha} \hat{V}\right] \\
& +\mathcal{O}\left(\hbar^{2}\right) .
\end{aligned}
$$

The kinetic equations (60) are the main result of the present paper. For the sake of notational convenience, we will omit the hat in the following.

In order to write the first kinetic equation (60) in terms of the distribution functions, we define

$$
V(x, p) \equiv \frac{2}{(2 \pi \hbar)^{3}} \sum_{e s} \theta\left(e p^{0}\right) f_{s}^{e}(x, e \mathbf{p}),
$$

where $f_{s}^{ \pm}=f_{s}^{(0) \pm}+\hbar f_{s}^{(1) \pm}$. Because of the theta function, the support of the distribution function for antiparticles is different from the one for particles. Thus, these distribution functions have to fulfill the first equation (60) separately [45]. Then, using Eqs. (30), (51), and (61), the first equation (60) can be written as 


$$
\begin{aligned}
0= & \sum_{s} \delta\left(p^{2}-m^{2}\right)\left[p \cdot \nabla^{(0)}+s \frac{\hbar}{4}\left(\partial_{x}^{\mu} F^{\nu \rho}\right) \partial_{p \mu} \Sigma_{\nu \rho}^{(0)}\right] \\
& \times \theta\left( \pm p_{0}\right) f_{s}^{ \pm} .
\end{aligned}
$$

To conclude this section, we remark that the terms containing the derivative of the delta function, although they do not contribute to the kinetic equations, lead to a modification of the on-shell condition of the components of the Wigner function. Noting that

$$
\begin{aligned}
& \delta\left(p^{2}-m^{2}-s \frac{\hbar}{2} F^{\mu \nu} \Sigma_{\mu \nu}^{(0)}\right) \\
& \quad=\delta\left(p^{2}-m^{2}\right)-s \frac{\hbar}{2} F^{\mu \nu} \Sigma_{\mu \nu}^{(0)} \delta^{\prime}\left(p^{2}-m^{2}\right)+\mathcal{O}\left(\hbar^{2}\right),
\end{aligned}
$$

we can for instance combine Eqs. (37) and (41) and use Eqs. (29) and (30) to obtain to order $\mathcal{O}(\hbar)$

$$
\begin{aligned}
\mathcal{F}= & \mathcal{F}^{(0)}+\hbar \mathcal{F}^{(1)} \\
= & \frac{2}{(2 \pi \hbar)^{3}} m \sum_{s} \delta\left(p^{2}-m^{2}-s \frac{\hbar}{2} F^{\mu \nu} \Sigma_{\mu \nu}^{(0)}\right) \\
& \times\left[\theta\left(p^{0}\right) f_{s}^{+}+\theta\left(-p^{0}\right) f_{s}^{-}\right] .
\end{aligned}
$$

Thus, to first order in $\hbar$ the on-shell condition is modified to

$$
p^{2}=m_{s}^{2} \equiv m^{2}+s \frac{\hbar}{2} F^{\mu \nu} \Sigma_{\mu \nu}^{(0)} .
$$

In the following, we discuss the massless limit and the classical case, as well as some consequences for global equilibrium and fluid dynamics.

\section{MASSLESS LIMIT}

In this section, we explain how to obtain the massless limit of the currents $\mathcal{V}_{\mu}$ and $\mathcal{A}_{\mu}$, cf. Eqs. (38) and (44). The crucial step is to replace the dipole-moment tensor (31) for $m \neq 0$ by the corresponding one for $m=0$. Obviously, this cannot be achieved simply by taking the limit $m \rightarrow 0$ in Eq. (31).

For massive particles, the dipole-moment tensor as well as the particle's position are uniquely defined in the rest frame. The Pauli-Lubanski operator is defined as [54]

$$
\hat{N}^{\mu}=-\frac{1}{2 m} \epsilon^{\mu \nu \rho \sigma} \sigma_{\nu \rho} \hat{P}_{\sigma},
$$

where $\hat{P}^{\mu} \equiv i \hbar D^{\mu}$ is the (kinetic) momentum operator. In the rest frame, the Pauli-Lubanski operator fulfills the commutation relations of an angular momentum. Let $\psi, \bar{\psi}$ be solutions of the Dirac equation (3). Then the dipolemoment tensor $\Sigma^{\mu \nu} \equiv \bar{\psi} \sigma^{\mu \nu} \psi$ fulfills $p_{\mu} \Sigma^{\mu \nu}=0$, where $p_{\mu}$ is the eigenvalue of $\hat{P}_{\mu}$. Thus,

$$
\Sigma^{\mu \nu}=-\frac{1}{m} \epsilon^{\mu \nu \alpha \beta} p_{\alpha} n_{\beta},
$$

with $n_{\beta}=\bar{\psi} \hat{N}_{\beta} \psi$. This agrees with Eq. (31), if $\psi=u$ or $v$.

On the classical level, $\Sigma^{\mu \nu}$ is the intrinsic angularmomentum tensor about the center of mass. In a relativistic theory, the center of mass of a particle is frame-dependent. In order to have a frame-independent definition of $\Sigma^{\mu \nu}$, one requires $p_{\mu} \Sigma^{\mu \nu}=0$ as a gauge condition. This requirement identifies the dipole-moment tensor (67) as the intrinsic angular-momentum tensor about the center of mass in the rest frame of the particle [55].

For massless particles there is no rest frame, thus both the position (in the classical case the center of momentum) and the dipole-moment tensor can at first be defined in an arbitrary frame, which makes them frame-dependent. For massless particles, the polarization vector $n^{\mu}$ is always parallel to the momentum $p^{\mu}$. Thus, the requirement $p_{\mu} \Sigma^{\mu \nu}=0$ can no longer be used as a gauge condition, since Eq. (67) automatically satisfies this constraint. [In the massless limit, one also needs to change the normalization of the spinors to $\bar{u} u=2|\mathbf{p}|$ [50].] If we choose the dipolemoment tensor to be defined in a frame characterized by a timelike four-vector $u^{\mu}$, we can choose the gauge condition $u^{\mu} \Sigma_{\mu \nu}=0$ [13]. Consequently, the frame vector $u^{\mu}$ must assume the role of $p^{\mu}$ in Eq. (67). Moreover, since $n^{\mu}$ and $p^{\mu}$ are parallel for massless particles, the momentum $p^{\mu}$ can assume the role of $n^{\mu}$ in Eq. (67). Finally, in order to obtain the massless case we need to replace the normalization factor $1 / m$ in Eq. (67). The energy of a massive particle in its rest frame is $p_{r f}^{0}=\sqrt{p^{2}}$. If the particle is on the massshell, this is equivalent to $p_{r f}^{0}=m$. The energy of a massless particle in the rest frame of $u^{\mu}$, however, is $p_{u}^{0}=p \cdot u$. Thus, it is natural to replace the normalization $1 / m$ in Eq. (67) by $1 /(p \cdot u)$. We emphasize that this replacement can only be done in the presence of a $\delta$-function which sets the rest-frame energy equal to the mass $m$. The explicit expression for the dipole-moment tensor in the massless case is then given by

$$
\Sigma_{u}^{\mu \nu}=-\frac{1}{p \cdot u} \epsilon^{\mu \nu \alpha \beta} u_{\alpha} p_{\beta},
$$

which agrees with the definition of the "spin tensor" in Ref. [13]. This tensor corresponds classically to the intrinsic angular momentum about the center of momentum as seen from the frame where $u^{\mu}=(1,0,0,0)$ and will have the quantum-mechanical properties of an angular-momentum operator in that frame.

With this knowledge, we can make the transition between the Wigner functions of massive and massless particles. For zero fermion mass, Eqs. (15) and (20) decouple. By defining right- and left-handed currents $J_{\chi}^{\mu} \equiv$ $\frac{1}{2}\left(\mathcal{V}^{\mu}+\chi \mathcal{A}^{\mu}\right), \chi= \pm$ for right-/left-handed particles, we have to order $\hbar$

$$
\frac{\hbar}{2}\left(\nabla^{\mu} J_{\chi}^{\nu}-\nabla^{\nu} J_{\chi}^{\mu}\right)=\chi \epsilon^{\mu \nu \alpha \beta} p_{\alpha} J_{\chi, \beta} .
$$


These equations have been solved in Refs. [15,17,18], with the result

$$
J_{\chi}^{\mu}=\delta\left(p^{2}\right)\left(p^{\mu}+\chi \frac{\hbar}{2} \Sigma_{u}^{\mu \nu} \nabla_{\nu}\right) f_{\chi}+\chi \hbar \tilde{F}^{\mu \nu} p_{\nu} \delta^{\prime}\left(p^{2}\right) f_{\chi},
$$

where $f_{\chi}$ is the distribution function for right-/left-handed fermions and $u^{\mu}$ is the four-velocity of an arbitrary frame. We remark that in the massive case, $s$ describes "spin up" or "spin down," which corresponds to positive or negative helicity in the massless limit $\left(m n_{\mu} \rightarrow p_{\mu}\right)$. On the other hand, the currents above are defined for given chirality $\chi$. Since helicity and chirality are identical for massless particles, but opposite for massless antiparticles, the relation between chirality $\chi$ and spin/helicity $s$ is $\chi=e s$ with $e= \pm$ representing particles/antiparticles.

To obtain the massless limit of our solutions, we replace the massive dipole-moment tensor by the massless one, $\Sigma^{(0) \mu \nu} \rightarrow \Sigma_{u}^{\mu \nu}$. In order to obtain the vector current for the massless case from Eq. (44), we need to consider the term $\sim \nabla^{(0) \nu} \Sigma_{\mu \nu}^{(0)}$. We first pull the constant factor $1 / m$ out of the derivative and then replace $\delta\left(p^{2}-m^{2}\right) / m=\delta\left(p^{2}-m^{2}\right) /$ $\sqrt{p^{2}} \rightarrow \delta\left(p^{2}\right) /(p \cdot u)$. Finally, replacing $p^{\mu} / m \rightarrow u^{\mu}$, $m n^{\mu} \rightarrow p^{\mu}$ in this term we find

$$
\begin{aligned}
\mathcal{V}_{\mu, m=0}^{(1)}= & \delta\left(p^{2}\right)\left[p_{\mu} V^{(1)}+\frac{1}{2 p \cdot u} \epsilon_{\mu \nu \alpha \beta} \nabla^{(0) \nu} p^{\alpha} u^{\beta} A^{(0)}\right] \\
& -\left[\frac{1}{2} p_{\mu} F^{\alpha \beta} \Sigma_{u, \alpha \beta}+\Sigma_{u, \mu \nu} F^{\nu \alpha} p_{\alpha}\right] A^{(0)} \delta^{\prime}\left(p^{2}\right) .
\end{aligned}
$$

In Ref. [15] the frame-vector $u_{\mu}$ is assumed to be independent of space-time coordinates. In order to compare to the solution found in that reference, we adopt the same assumption. Evaluating the derivatives, contracting the $\epsilon$-tensors, and using $p^{2} \delta^{\prime}\left(p^{2}\right)=-\delta\left(p^{2}\right)$, we find from Eqs. (38) and (71)

$$
\begin{aligned}
\mathcal{V}_{m=0}^{\mu}= & \delta\left(p^{2}\right)\left[p^{\mu} V+\frac{\hbar}{2} \Sigma_{u}^{\mu \nu} \nabla_{\nu}^{(0)} A^{(0)}\right] \\
& +\hbar \tilde{F}^{\mu \nu} p_{\nu} A^{(0)} \delta^{\prime}\left(p^{2}\right)+\mathcal{O}\left(\hbar^{2}\right),
\end{aligned}
$$

where $V=V^{(0)}+\hbar V^{(1)}$. Note that $V^{(1)}$ depends on the frame vector $u^{\mu}$ such that the whole expression (72) is frame independent $[13,17,20]$. To obtain the axial-vector current in the massless case from Eqs. (38) and (44), we note that the general solution of Eq. (42) reads

$$
\bar{\Sigma}_{\mu \nu}^{(1)}=\Sigma_{v, \mu \nu} A^{(1)}+\frac{1}{2 p \cdot u}\left(u_{\nu} \nabla_{\mu}^{(0)}-u_{\mu} \nabla_{\nu}^{(0)}\right) V^{(0)},
$$

where the first and second terms depend on arbitrary timelike unit vectors $u^{\mu}$ and $v^{\mu}$, respectively. Here, one makes use of the first equation (47) to see that the constraint (42) is fulfilled. Inserting Eq. (73) into Eq. (44), and replacing the zeroth order dipole-moment tensor $\Sigma_{\mu \nu}^{(0)}$ by $\Sigma_{u, \mu \nu}$, we find

$$
\begin{aligned}
\mathcal{A}_{m=0}^{\mu}= & \delta\left(p^{2}\right)\left[p^{\mu} A+\frac{\hbar}{2} \Sigma_{u}^{\mu \nu} \nabla_{\nu}^{(0)} V^{(0)}\right] \\
& +\hbar \tilde{F}^{\mu \nu} p_{\nu} V^{(0)} \delta^{\prime}\left(p^{2}\right)+\mathcal{O}\left(\hbar^{2}\right),
\end{aligned}
$$

where $A \equiv A^{(0)}+\hbar A^{(1)}$, with $A^{(1)}$ dependent on $u^{\mu}$. Note that, in order for the above axial current to be frameindependent, the function $A^{(1)}$ cannot depend on $v^{\mu}$. Adding and subtracting Eqs. (72) and (74), we recover the result (70). Acting with $\nabla_{\mu}$ on this equation, one can derive the chiral kinetic theory of Refs. [15-20].

\section{COMPARISON TO THE CLASSICAL CASE}

In this section, we show that Eq. (62) gives rise to the first and second Mathisson-Papapetrou-Dixon (MPD) equations [51,56] as well as to the BMT equation [53], which were derived for classical, extended, spinning particles with nonvanishing dipole moment. Comparing Eq. (62) to the generic form of the collisionless relativistic Boltzmann-Vlasov equation $[56,57]$

$$
p \cdot \partial_{x} f_{s}+m \partial_{p \mu}\left(F_{s}^{\mu} f_{s}\right)=0,
$$

where $f_{s}$ is the distribution function, $F_{s}^{\mu}=d p^{\mu} / d \tau$ is the external force, $p^{\mu}=m d x^{\mu} / d \tau$ and $\tau$ the world-line parameter, we find that in our case

$$
F_{s}^{\mu}=\frac{1}{m}\left[F^{\mu \nu} p_{\nu}+s \frac{\hbar}{4}\left(\partial_{x}^{\mu} F^{\nu \rho}\right) \Sigma_{\nu \rho}^{(0)}\right],
$$

i.e., the external force is given as the sum of the Lorentz force and the Mathisson force. This is the first MPD equation [51,56]. In Refs. [51,56], the kinetic equation for particles with classical dipole moment $m^{\mu \nu}$ was derived. Our results agree with those, setting

$$
m_{\mu \nu} \rightarrow g \mu_{B} \frac{s}{2} \Sigma_{\mu \nu}^{(0)},
$$

with Bohr's magneton $\mu_{B} \equiv \mathrm{e} \hbar /(2 m)$, where $\mathfrak{e}$ is the electric charge, and the gyromagnetic ratio $g=2$, as expected for Dirac particles with spin $1 / 2$.

The evolution of the dipole-moment tensor is given by the third equation (47), which can be rewritten as

$$
m \dot{\Sigma}_{\mu \nu}^{(0)}=F_{[\mu}^{\alpha} \Sigma_{\nu] \alpha}^{(0)},
$$

where we used

$$
\dot{\Sigma}_{\mu \nu}^{(0)} \equiv\left(\dot{x}^{\alpha} \partial_{x \alpha}+\dot{p}^{\alpha} \partial_{p \alpha}\right) \Sigma_{\mu \nu}^{(0)}
$$

with $F_{s}^{\mu}$ given by Eq. (76) to zeroth order. Equation (78) is identical to the second MPD equation $[51,56]$. Using Eq. (31), we obtain 


$$
m \dot{\Sigma}_{\mu \nu}^{(0)}=-\epsilon_{\mu \nu \alpha \beta}\left(p^{\alpha} \dot{n}^{(0) \beta}-\frac{1}{m} F^{\lambda \alpha} p_{\lambda} n^{(0) \beta}\right) .
$$

Inserting Eq. (78) and contracting with $\epsilon^{\rho \sigma \mu \nu}$ yields

$p_{\rho}\left(m \dot{n}_{\sigma}^{(0)}+F_{\sigma}^{\mu} n_{\mu}^{(0)}\right)-p_{\sigma}\left(m \dot{n}_{\rho}^{(0)}+F_{\rho}^{\mu} n_{\mu}^{(0)}\right)=0$

Contracting with $p^{\rho}$ and using Eq. (76) to zeroth order in $\hbar$, we conclude that

$$
m \dot{n}_{\mu}^{(0)}=F_{\mu \nu} n^{(0) \nu}
$$

This is the BMT equation for classical spin precession in an electromagnetic field [53].

\section{GLOBAL EQUILIBRIUM}

Equation (62) determines the single-particle distribution function $f_{s}^{ \pm}$in a general nonequilibrium state. A special solution is obtained in global equilibrium, which we will consider in this section.

A necessary condition for equilibrium is vanishing entropy production. Assuming the standard form of the collision term, the distribution function in equilibrium must have the form $[13,56]$

$$
f_{s}^{e q}=\left(e^{g_{s}}+1\right)^{-1}
$$

with $g_{s}$ being a linear combination of the collisional invariants, namely, charge, kinetic momentum $p^{\mu}$, and total angular momentum

$$
J_{s}^{\mu \nu}=L^{\mu \nu}+s \frac{\hbar}{2} \Sigma^{(0) \mu \nu}+\mathcal{O}\left(\hbar^{2}\right)
$$

which is the sum of orbital angular momentum $L^{\mu \nu}=$ $x^{[\mu} p^{\nu]}$ and spin angular momentum, which to first order is given by the dipole-moment tensor $s \frac{\hbar}{2} \Sigma^{(0) \mu \nu}$. (Also the canonical momentum $\pi^{\mu}$ is conserved in a collision and could be used instead of the kinetic momentum. Here, we will at first use the kinetic momentum, since it is independent of space-time coordinates, as well as gaugeindependent.) Thus,

$$
g_{s}=p \cdot b(x)+a_{s}(x)+\frac{1}{2} \Omega_{\mu \nu}(x) J_{s}^{\mu \nu}
$$

Here, $b_{\mu}(x), a_{s}(x)$, and $\Omega_{\mu \nu}(x)$ are Lagrangian multipliers, which can depend on $x$. Since $J_{s}^{\mu \nu}$ is antisymmetric, the symmetric part of $\Omega_{\mu \nu}$ can be dropped without loss of generality.

Let us consider the case of global equilibrium with rigid rotation. Using Eqs. (84) and (85) can be written as

$$
g_{s}=p \cdot \beta(x)+a_{s}(x)+s \frac{\hbar}{4} \Omega_{\mu \nu}(x) \Sigma^{(0) \mu \nu},
$$

where $\beta_{\mu}(x) \equiv b_{\mu}(x)+\Omega_{\nu \mu}(x) x^{\nu}$. In global equilibrium, the Boltzmann equation (62) needs to be fulfilled. From the part of Eq. (62) proportional to the derivative of $f_{s}^{e q}$ we obtain

$$
\begin{aligned}
0= & \left\{p^{\mu} \partial_{x \mu}+\left[F^{\mu \nu} p_{\nu}+s \frac{\hbar}{4} \Sigma_{\nu \lambda}^{(0)}\left(\partial_{x}^{\mu} F^{\nu \lambda}\right)\right] \partial_{p \mu}\right\} g_{s} \\
= & p^{\mu}\left[\partial_{x \mu} a_{s}(x)+F_{\nu \mu} \beta^{\nu}(x)\right]+p^{\mu} p^{\nu} \partial_{x \mu} \beta_{\nu}(x) \\
& +s \frac{\hbar}{4} \Sigma^{(0) \rho \sigma} p \cdot \partial_{x} \Omega_{\rho \sigma}(x) \\
& +s \frac{\hbar}{4}\left[\Sigma^{(0) \rho \sigma} \beta(x) \cdot \partial_{x} F_{\rho \sigma}+\Sigma^{(0) \mu[\sigma} F_{\mu}^{\rho]} \Omega_{\rho \sigma}(x)\right],
\end{aligned}
$$

where we used Eq. (47). This equation is fulfilled, if

$$
\begin{aligned}
\partial_{x \mu} \beta_{\nu}+\partial_{x \nu} \beta_{\mu} & =0, \\
\partial_{x \mu} a_{s}(x) & =F_{\mu \nu} \beta^{\nu}(x), \\
\partial_{x \mu} \Omega_{\lambda \nu}(x) & =0,
\end{aligned}
$$

which makes the terms in the first and second line of Eq. (88) vanish. The terms in the third line of Eq. (87) can be shown to vanish if $b_{\mu}$ is constant, since then $\Omega_{\mu \nu}$ is equal to the thermal vorticity, i.e.,

$$
\Omega_{\mu \nu}=\omega_{\mu \nu} \equiv \frac{1}{2}\left(\partial_{x \mu} \beta_{\nu}-\partial_{x \nu} \beta_{\mu}\right) .
$$

For the proof, one also employs the relation

$$
\beta^{\alpha} \partial_{x \alpha} F_{\mu \nu}-F_{\alpha \mu} \partial_{x \nu} \beta^{\alpha}+F_{\alpha \nu} \partial_{x \mu} \beta^{\alpha}=0,
$$

which can be proven with the help of the homogeneous Maxwell equations and Eq. (88). These equilibrium conditions agree with those found in the classical case [56] and those using covariant statistical mechanics [58]. Note that the second equation (88) implies that, in the rest frame of $\beta^{\mu}$, an electric field is cancelled by a gradient in $a_{s}$. It is amusing to note that, without electromagnetic fields, the tensor $\Omega_{\mu \nu}$ does not need to be equal to the thermal vorticity.

We introduce the Lie derivative of $\mathbb{A}_{\mu}$ along the direction of $\beta^{\lambda}$ as

$$
\mathcal{L}_{\beta} \mathbb{A}_{\mu}(x) \equiv \beta(x) \cdot \partial_{x} \mathbb{A}_{\mu}(x)-\mathbb{A}(x) \cdot \partial_{x} \beta_{\mu}(x) .
$$

Choosing a gauge in which $\mathcal{L}_{\beta} \mathbb{A}_{\mu}=0$, we can rewrite Eq. (88) as

$$
\partial_{x \mu}\left[a_{s}(x)-\mathbb{A}(x) \cdot \beta(x)\right]=0 .
$$

Defining

$$
-\beta(x) \mu_{s}(x) \equiv a_{s}(x)-\mathbb{A}(x) \cdot \beta(x)=\mathrm{const},
$$

the function $g_{s}$ becomes 


$$
g_{s}=\beta \pi \cdot U-\beta \mu_{s}+s \frac{\hbar}{4} \Sigma^{(0) \mu \nu} \omega_{\mu \nu}
$$

Here, $\pi_{\mu} \equiv p_{\mu}+\mathbb{A}_{\mu}$ is the canonical momentum, $U^{\mu}$ is the fluid velocity, $\beta \equiv 1 / T$ is the inverse temperature, and $\mu_{s}$ is the chemical potential for particles with spin $s$ (for antiparticles, we need to reverse the sign of the chemical potential). This form of $g_{s}$, and thus the distribution function $f_{s}^{e q}$, agrees in the massless and field-free limit to the one suggested in Ref. [13]. Moreover, recalling the definition (A12), (A13) of the dipole-moment tensor one can prove that the distribution function agrees with the one proposed in Ref. [33] to first order in $\hbar$ if $\mu_{+}=\mu_{-}$and if the electromagnetic field vanishes.

The part of Eq. (62) which is proportional to $f_{s}^{ \pm}$vanishes if $\mu_{+}=\mu_{-}$to zeroth order in $\hbar$. In the presence of a spin imbalance, $\Delta \mu \equiv \mu_{+}-\mu_{-} \neq 0$, it only vanishes if

$$
\left(\partial_{x}^{\lambda} F^{\nu \rho}\right)\left(\partial_{p \lambda} \Sigma_{\nu \rho}^{(0)}\right)=0 .
$$

The reason that global equilibrium with spin imbalance can in general not be realized for massive particles is that in this case the axial-vector current is only conserved if the pseudoscalar function $\mathcal{P}=0$, see also Eq. (12).

To zeroth order in $\hbar$, the distribution function is given by

$$
f_{s}^{(0)}=\left(e^{g_{s}^{(0)}}+1\right)^{-1},
$$

with

$$
g_{s}^{(0)}=\beta\left(\pi \cdot U-\mu_{s}\right)
$$

We define the dual thermal vorticity tensor as $\tilde{\omega}_{\mu \nu} \equiv$ $\frac{1}{2} \epsilon_{\mu \nu \alpha \beta} \omega^{\alpha \beta}$. Now we calculate the vector current by inserting the distribution function (83) into the equation for $\mathcal{V}_{\mu}=$ $\mathcal{V}_{\mu}^{(0)}+\hbar \mathcal{V}_{\mu}^{(1)}$, cf. Eqs. (38), (44). With

$$
\begin{aligned}
\nabla_{\nu}^{(0)} f_{s}^{(0)} & =\frac{\partial f_{s}^{(0)}}{\partial g_{s}^{(0)}}\left(\pi^{\alpha} \partial_{x \nu} \beta_{\alpha}+\beta^{\alpha} \partial_{x \nu} \mathbb{A}_{\alpha}-F_{\nu \alpha} \beta^{\alpha}\right) \\
& =\frac{\partial f_{s}^{(0)}}{\partial g_{s}^{(0)}} p^{\alpha} \omega_{\nu \alpha},
\end{aligned}
$$

where we used $\mathcal{L}_{\beta} \mathrm{A}_{\alpha}=0$, Taylor-expanding

$$
f_{s}^{e q}=f_{s}^{(0)}+\frac{s \hbar}{4} \Sigma_{\mu \nu}^{(0)} \omega^{\mu \nu} \frac{\partial f_{s}^{(0)}}{\partial g_{s}^{(0)}}+\mathcal{O}\left(\hbar^{2}\right),
$$

and noting that

$$
\begin{aligned}
& \delta\left(p^{2}-m^{2}\right)\left(\Sigma^{(0) \mu \nu} p^{\rho} \omega_{\nu \rho}+\frac{1}{2} \Sigma^{(0) \rho \nu} p^{\mu} \omega_{\rho \nu}\right) \\
& =-\delta\left(p^{2}-m^{2}\right) m \tilde{\omega}^{\mu \nu} n_{\nu}
\end{aligned}
$$

where we used $p \cdot n=0$ and $\delta\left(p^{2}-m^{2}\right) p^{2}=\delta\left(p^{2}-m^{2}\right) m^{2}$, we find

$$
\begin{aligned}
\mathcal{V}^{\mu}= & \frac{2}{(2 \pi \hbar)^{3}} \sum_{s}\left[\delta\left(p^{2}-m^{2}\right)\left(p^{\mu}-m s \frac{\hbar}{2} \tilde{\omega}^{\mu \nu} n_{\nu} \frac{\partial}{\partial g_{s}^{(0)}}\right)\right. \\
& +m s \hbar \tilde{F}^{\mu \nu} n_{\nu} \delta^{\prime}\left(p^{2}-m^{2}\right) \\
& \left.-\frac{s \hbar}{2 m} \delta\left(p^{2}-m^{2}\right) \epsilon^{\mu \nu \alpha \beta} p_{\alpha}\left(\nabla_{\nu}^{(0)} n_{\beta}\right)\right] \\
& \times\left[\theta\left(p^{0}\right) f_{s}^{(0)+}+\theta\left(-p^{0}\right) f_{s}^{(0)-}\right]+\mathcal{O}\left(\hbar^{2}\right) .
\end{aligned}
$$

The current given by Eq. (101) contains contributions which are not parallel to $p^{\mu}$. To first order in $\hbar$, particles are not transported parallel to their momenta. The term containing $\tilde{F}^{\mu \nu}$ in Eqs. (101) is caused by off-shell effects and describes the vector current induced by electromagnetic fields, which yields the analogue of the CME in the case of nonzero mass. On the other hand, the term containing $\tilde{\omega}^{\mu \nu}$ describes the current induced by vorticity and thus gives the analogue of the CVE.

We furthermore calculate the axial-vector current. In order to do so, it is convenient to decompose the tensor $\bar{\Sigma}_{\mu \nu}^{(1)}$ introduced in Eq. (41) in the following way,

$$
\bar{\Sigma}_{\mu \nu}^{(1)} \equiv \frac{1}{2} \chi_{\mu \nu}+\Xi_{\mu \nu}
$$

The tensor $\Xi_{\mu \nu}$ is antisymmetric and satisfies $p^{\mu} \Xi_{\mu \nu}=0$. On the other hand, $\chi_{\mu \nu}$ represents the dipole moment induced by the gradients of the distribution function since, according to Eq. (42), it satisfies

$$
p^{\nu} \chi_{\mu \nu}=\nabla_{\mu}^{(0)} V^{(0)} .
$$

Inserting $V^{(0)}$ into Eq. (103) and using Eq. (98) we can derive the following constraint for $\chi_{\mu \nu}$,

$$
p^{\nu} \chi_{\mu \nu}=p^{\nu} \omega_{\mu \nu} V^{(0) \prime},
$$

where we adopted the short-hand notation

$$
V^{(0) \prime} \equiv \frac{2}{(2 \pi \hbar)^{3}} \sum_{s} \frac{\partial}{\partial g_{s}^{(0)}}\left[\theta\left(p^{0}\right) f_{s}^{(0)+}+\theta\left(-p^{0}\right) f_{s}^{(0)-}\right] .
$$

The most general solution of Eq. (104) can be written as

$$
\chi_{\mu \nu}=\left[\kappa_{1} \omega_{\mu \nu}-\frac{\kappa_{2}}{v \cdot p}\left(v_{\mu} \omega_{\nu \alpha}-v_{\nu} \omega_{\mu \alpha}\right) p^{\alpha}\right] V^{(0) \prime},
$$

where $\kappa_{1,2}$ are arbitrary coefficients which satisfy $\kappa_{1}+$ $\kappa_{2}=1$, and $v^{\mu}$ is an arbitrary vector such that $v \cdot p \neq 0$. Other possible terms which vanish when being contracted with the momentum are absorbed into $\Xi_{\mu \nu}$. 
The decomposition into $\chi_{\mu \nu}$ and $\Xi_{\mu \nu}$ is not unique, but allows for the transformations

$$
\begin{aligned}
& \Xi_{\mu \nu} \rightarrow \Xi_{\mu \nu}-\frac{C}{2}\left[\omega_{\mu \nu}+\frac{1}{v \cdot p}\left(v_{\mu} \omega_{\nu \alpha}-v_{\nu} \omega_{\mu \alpha}\right) p^{\alpha}\right] V^{(0) \prime}, \\
& \chi_{\mu \nu} \rightarrow \chi_{\mu \nu}+C\left[\omega_{\mu \nu}+\frac{1}{v \cdot p}\left(v_{\mu} \omega_{\nu \alpha}-v_{\nu} \omega_{\mu \alpha}\right) p^{\alpha}\right] V^{(0) \prime},
\end{aligned}
$$

with $C$ being an arbitrary function of $x$ and $p$. For any value of $\kappa_{2}$, we can apply the above transformation with $C \equiv \kappa_{2}$ to Eq. (106), which yields

$$
\chi_{\mu \nu}=\left(\kappa_{1}+\kappa_{2}\right) \omega_{\mu \nu} V^{(0) \prime}=\omega_{\mu \nu} V^{(0) \prime} .
$$

Thus, we can set $\kappa_{2}=0$ without loss of generality. In other words, it is always possible to isolate the contribution proportional to $\omega_{\mu \nu}$ in the decomposition for $\bar{\Sigma}_{\mu \nu}^{(1)}$. This decomposition will assume a physical meaning when looking at the kinetic equation for $\mathcal{S}_{\mu \nu}$.

Inserting Eq. (102) into Eq. (49), we obtain

$$
\begin{aligned}
0= & p \cdot \nabla^{(0)}\left(\frac{1}{2} \chi_{\mu \nu}+\Xi_{\mu \nu}\right)-F_{[\mu}^{\alpha}\left(\frac{1}{2} \chi_{\nu] \alpha}+\Xi_{\nu] \alpha}\right) \\
& +\frac{1}{2}\left(\partial_{x \alpha} F_{\mu \nu}\right) \partial_{p}^{\alpha} V^{(0)} .
\end{aligned}
$$

Noting that $p \cdot \nabla^{(0)} \chi_{\mu \nu}=0$ and using Eqs. (90) and (108), we find that the $\chi_{\mu \nu}$-dependent part vanishes and

$$
p \cdot \nabla^{(0)} \Xi_{\mu \nu}=F_{[\mu}^{\alpha} \Xi_{\nu] \alpha},
$$

which is the second MPD equation for $\Xi_{\mu \nu}$. This part of the dipole-moment corresponds, together with the zeroth-order dipole moment, to the classical spin precession in electromagnetic fields.

We now derive from Eq. (44) the full axial-vector part of the Wigner function up to first order in $\hbar$, i.e.,

$$
\begin{aligned}
\mathcal{A}^{\mu}= & \frac{2}{(2 \pi \hbar)^{3}} \sum_{s}\left[\delta\left(p^{2}-m^{2}\right)\left(s m n^{(0) \mu}-\frac{\hbar}{2} \tilde{\omega}^{\mu \nu} p_{\nu} \frac{\partial}{\partial g_{s}^{(0)}}\right)\right. \\
& \left.+\hbar \tilde{F}^{\mu \nu} p_{\nu} \delta^{\prime}\left(p^{2}-m^{2}\right)\right]\left[\theta\left(p^{0}\right) f_{s}^{(0)+}+\theta\left(-p^{0}\right) f_{s}^{(0)-}\right] \\
& -\frac{\hbar}{2} \epsilon^{\mu \nu \alpha \beta} p_{\nu} \Xi_{\alpha \beta} \delta\left(p^{2}-m^{2}\right)+\mathcal{O}\left(\hbar^{2}\right) .
\end{aligned}
$$

By looking at the different terms in Eq. (111), we identify three contributions to the axial-vector current in the massive case. The first term in the first line and the term in the last line describe the spin precession in the presence of an electromagnetic field according to the BMT equation. We remark that the function $\Xi_{\mu \nu}$ is not specified and has to be determined through Eq. (110). The second term in the first line gives rise to the axial current in the direction of the vorticity, which is the analogue of the axial chiral vortical effect (ACVE). Finally, the term in the second line describes the axial current along the magnetic field, which is the analogue of the chiral separation effect (CSE). These terms are analogous to those found in Refs. [35,41,59].

\section{FLUID-DYNAMICAL EQUATIONS}

In this section, we present the equations of motion of the fluid-dynamical variables, i.e., of the net particle-number current and the energy-momentum tensor. We also give an equation for the spin tensor, which supplements these equations in the case of spin-1/2 particles.

The net particle-number current is defined as

$$
J^{\mu}(x) \equiv\left\langle: \bar{\psi}(x) \gamma^{\mu} \psi(x):\right\rangle=\int d^{4} p \mathcal{V}^{\mu}(x, p) .
$$

Inserting the zeroth- and first-order solutions (38), (44) into Eq. (112) we obtain

$$
\begin{aligned}
J^{\mu}= & \int d P p^{\mu}\left[V^{(0)}+\hbar V^{(1)}\right]+\frac{\hbar}{2} \partial_{x \nu} \int d P \Sigma^{(0) \mu \nu} A^{(0)} \\
& +\frac{\hbar}{4} F_{\alpha \beta} \int d P \partial_{p}^{\mu}\left[\Sigma^{(0) \alpha \beta} A^{(0)}\right]
\end{aligned}
$$

where $d P \equiv d^{4} p \delta\left(p^{2}-m^{2}\right)$.

Equation (16) represents the conservation law for the vector component of the Wigner function. Integrating this equation over kinetic 4-momentum, we immediately obtain the conservation law for the net particle-number current,

$\partial_{x \mu} J^{\mu}(x)=\int d^{4} p\left[\nabla_{\mu}+j_{0}(\Delta) F_{\mu \nu} \partial_{p}^{\nu}\right] \mathcal{V}^{\mu}(x, p)=0$,

where we assumed that $F^{\mu \nu}$ is independent of $p^{\nu}$ and $\mathcal{V}^{\mu}$ vanishes sufficiently rapidly for large momenta, which ensures the vanishing of a boundary term.

The Lagrangian operator for a Dirac spinor in an electromagnetic field is [45]

$$
\mathcal{L}=\bar{\psi}\left[i \frac{\hbar}{2} \gamma \cdot\left(\vec{D}-\overleftarrow{D}^{\dagger}\right)-m\right] \psi-\frac{1}{4} F^{\mu \nu} F_{\mu \nu} .
$$

From the Lagrangian we can derive the canonical energymomentum tensor as follows,

$$
\begin{aligned}
T^{\mu \nu}= & \left\langle: \frac{\partial \mathcal{L}}{\partial\left(\partial_{x \mu} \psi\right)} \partial_{x}^{\nu} \psi+\partial_{x}^{\nu} \bar{\psi} \frac{\partial \mathcal{L}}{\partial\left(\partial_{x \mu} \bar{\psi}\right)}\right. \\
& \left.+\frac{\partial \mathcal{L}}{\partial\left(\partial_{x \mu} \mathbb{A}_{\alpha}\right)} \partial_{x}^{\nu} \mathbb{A}_{\alpha}-g^{\mu \nu} \mathcal{L}:\right\rangle \\
= & T_{\text {mat }}^{\mu \nu}+T_{\mathrm{int}}^{\mu \nu}+T_{\mathrm{em}}^{\mu \nu},
\end{aligned}
$$


where we have separated the total energy-momentum tensor into three parts: the gauge-invariant matter part $T_{\text {mat }}^{\mu \nu}$, the part containing the interaction between gauge potential and matter current, $T_{\text {int }}^{\mu \nu}$, and the electromagnetic part $T_{\mathrm{em}}^{\mu \nu}$,

$$
\begin{aligned}
& T_{\text {mat }}^{\mu \nu}=\frac{\hbar}{2}\left\langle: \bar{\psi} \gamma^{\mu}\left(i \vec{D}^{\nu}-i \overleftarrow{D}^{\dagger \nu}\right) \psi:\right\rangle=\int d^{4} p p^{\nu} \mathcal{V}^{\mu}, \\
& T_{\text {int }}^{\mu \nu}=\mathbb{A}^{\nu}\left\langle: \bar{\psi} \gamma^{\mu} \psi:\right\rangle=\mathbb{A}^{\nu} \int d^{4} p \mathcal{V}^{\mu}, \\
& T_{\mathrm{em}}^{\mu \nu}=\frac{1}{4} g^{\mu \nu} F^{\alpha \beta} F_{\alpha \beta}-F^{\mu \alpha} \partial_{x}^{\nu} \mathbb{A}_{\alpha} .
\end{aligned}
$$

Note that none of these are in general symmetric under $\mu \leftrightarrow \nu$. The total energy-momentum tensor is conserved $\partial_{x \nu} T^{\mu \nu}=0$, which can be checked using the Dirac and Maxwell equations. However, the matter part is not conserved,

$$
\partial_{x \mu} T_{\text {mat }}^{\mu \nu}(x)=F^{\nu \alpha}(x) J_{\alpha}(x) .
$$

This equation can be derived by acting $\partial_{x \mu}$ on $\mathcal{V}^{\mu}$ in the definition of $T_{\text {mat }}^{\mu \nu}$, cf. first equation (117), then using Eq. (16), and finally integrating by parts. Inserting Eqs. (38) and (44) into the energy-momentum tensor, we get

$$
\begin{aligned}
T_{\text {mat }}^{\mu \nu}= & \int d P p^{\mu} p^{\nu}\left[V^{(0)}+\hbar V^{(1)}\right]+\frac{\hbar}{2} \partial_{x \alpha} \int d P p^{\nu} \Sigma^{(0) \mu \alpha} A^{(0)} \\
& +\frac{\hbar}{4} g^{\mu \nu} F^{\alpha \beta} \int d P \Sigma_{\alpha \beta}^{(0)} A^{(0)}-\frac{\hbar}{2} F_{\alpha}^{\nu} \int d P \Sigma^{(0) \mu \alpha} A^{(0)} \\
& +\frac{\hbar}{4} F^{\alpha \beta} \int d P p^{\mu} \partial_{p}^{\nu}\left[\Sigma_{\alpha \beta}^{(0)} A^{(0)}\right] .
\end{aligned}
$$

The total canonical angular momentum tensor is calculated as follows,

$$
\begin{aligned}
J^{\lambda, \mu \nu}= & x^{\mu} T^{\lambda \nu}-x^{\nu} T^{\lambda \mu}+\frac{\hbar}{4}\left\langle: \bar{\psi}\left\{\gamma^{\lambda}, \sigma^{\mu \nu}\right\} \psi:\right\rangle \\
& -\left(F^{\lambda \mu} \mathbb{A}^{\nu}-F^{\lambda \nu} \mathbb{A}^{\mu}\right) .
\end{aligned}
$$

The first two terms, $x^{\mu} T^{\lambda \nu}-x^{\nu} T^{\lambda \mu}$, can be interpreted as the orbital angular-momentum tensor. The remaining terms constitute the spin angular-momentum tensor, which can be further separated into a matter and a field part. The spin tensor of matter can be defined as [33]

$S_{\text {mat }}^{\lambda, \mu \nu}(x) \equiv \frac{1}{4}\left\langle: \bar{\psi}\left\{\gamma^{\lambda}, \sigma^{\mu \nu}\right\} \psi:\right\rangle=-\frac{1}{2} \epsilon^{\lambda \mu \nu \rho} \int d^{4} p \mathcal{A}_{\rho}(x, p)$.

With the help of Eq. (20) we find, to any order in $\hbar$,

$$
\hbar \partial_{x \lambda} S_{\text {mat }}^{\lambda, \mu \nu}(x)=T_{\text {mat }}^{\nu \mu}(x)-T_{\text {mat }}^{\mu \nu}(x),
$$

where we assumed that boundary terms vanish. Thus, the spin of matter is not conserved separately. To zeroth order in $\hbar, T_{\text {mat }}^{\mu \nu}$ is symmetric according to Eq. (119), thus both sides of Eq. (122) vanish. To first order in $\hbar$, both sides are nonzero. Inserting the zeroth-order Wigner function into Eq. (121) we obtain

$$
S_{\mathrm{mat}}^{(0) \lambda, \mu \nu}(x)=\frac{1}{2} \int d P\left[p^{\lambda} \Sigma^{(0) \mu \nu}+p^{\mu} \Sigma^{(0) \nu \lambda}-p^{\nu} \Sigma^{(0) \mu \lambda}\right] A^{(0)} .
$$

The above expressions for the energy-momentum and spin tensor emerge directly from Noether's theorem and thus correspond to the canonical ones. However, one can obtain different sets of tensors by applying pseudogauge transformations that keep the conservation laws for energymomentum and spin. It has been shown that using different sets of tensors related through this pseudogauge freedom is not equivalent and leads to different measurable quantities [40]. We should mention that a similar derivation of fluiddynamical equations of motion from the Wigner function for massless particles including the conservation of total angular momentum was carried out in Ref. [19].

Note that with Eq. (122), we can also prove that $\partial_{x \nu} T_{\text {mat }}^{\mu \nu}=F^{\mu \alpha} J_{\alpha}$, the form of the equation of motion for the matter energy-momentum tensor given in Refs. [60,61].

\section{CONCLUSIONS}

In this paper we have derived kinetic theory for massive spin-1/2 particles in an inhomogeneous electromagnetic field starting from the covariant formulation of the Wigner function. Carrying out an expansion in $\hbar$ and truncating it at first order, we found a general solution of the equations of motion. We showed how to consistently take the massless limit and demonstrated agreement with previous works, which describe the CME and CVE. One of the crucial results of our work is the derivation of the collisionless Boltzmann equation for particles that carry a dipole moment due to their spin. We also recovered well-known results in the classical limit. The external force acting on the particles is the sum of the Lorentz force and the Mathisson force, i.e., the first MPD equation. The time evolution of the dipole moment follows the second MPD equation, and the spin polarization precesses according to the BMT equation. Moreover, as an example, we studied the case of a rigidly rotating fluid in global equilibrium. In particular, we found the conditions that the Lagrange multipliers related to the conservation of charge, energy, momentum, and angular momentum have to satisfy in order for the distribution function to be a solution of the Boltzmann equation. Finally, fluid-dynamical equations of motion are provided, in which the spin tensor is included among the evolved densities.

A straightforward extension of this work could be the inclusion of a collision term into our generalized Boltzmann equation and the derivation of the equations of motion for dissipative relativistic magneto-hydrodynamics for spin- $1 / 2$ particles. This could be achieved using the method of 
moments, following Refs. [60,61], where this has already been done for spin- 0 particles. Another potential extension would be the derivation of a transport equation starting from the equal-time Wigner-function formalism [46].

\section{ACKNOWLEDGMENTS}

The authors thank F. Becattini, W. Florkowski, C. Greiner, K. Hattori, U. Heinz, X.-G. Huang, E. Molnár, L. Tinti, and $\mathrm{H}$. van Hees for enlightening discussions. The work of D.H.R., X.-1.S., E.S., and N.W. is supported by the Deutsche Forschungsgemeinschaft (DFG, German Research Foundation) through the Collaborative Research Center CRC-TR 211 "Stronginteraction matter under extreme conditions"-Project No. 315477589-TRR 211. D. H. R. acknowledges partial support by the High-end Foreign Experts project GDW20167100136 of the State Administration of Foreign Experts Affairs of China. X.-1. S. is supported in part by China Scholarship Council. E. S. acknowledges support by Bundesministerium für Bildung und Forschung (BMBF) "Verbundprojekt: 05P2015ALICE at High Rate," and BMBF "Forschungsprojekt: 05P2018 - Ausbau von ALICE am LHC (05P18RFCA1)". Q. W. is supported in part by the 973 program under Grant No. 2015CB856902 and by National Natural Science Foundation of China (NSFC) under Grant No. 11535012.

Note added.-After completion of this work, we became aware of a related study [62], where kinetic equations for massive fermions were derived using the covariant Wignerfunction approach. Other related work, which appeared after the submission of this paper, can be found in Refs. $[63,64]$.

\section{APPENDIX A: DIAGONAL SPIN BASIS}

In this Appendix, we show how to diagonalize the distribution function by choosing the spin quantization direction along the polarization direction. The axial-vector current which one obtains directly from Eq. (22) reads

$$
\begin{aligned}
\mathcal{A}_{\mu}^{(0)}(x, p)= & \frac{1}{(2 \pi \hbar)^{3}} \delta\left(p^{2}-m^{2}\right) \\
& \times \sum_{r s}\left[\theta\left(p^{0}\right) \bar{u}(\mathbf{p}, s) \gamma_{\mu} \gamma^{5} u(\mathbf{p}, r) f_{r s}^{(0)+}(x, \mathbf{p})\right. \\
& \left.-\theta\left(-p^{0}\right) \bar{v}(-\mathbf{p}, r) \gamma_{\mu} \gamma^{5} v(-\mathbf{p}, s) f_{s r}^{(0)-}(x,-\mathbf{p})\right] .
\end{aligned}
$$

The distribution functions $f^{(0) \pm}$ are Hermitian matrices in spin space and can thus be diagonalized by a unitary transformation [50]. Since the Pauli matrices $\sigma^{i}$ together with the unit matrix are a basis of the space of Hermitian $(2 \times 2)$ matrices, the distribution functions can be written as $[37,65]$

$$
f^{(0) e}=a^{e}+\mathbf{b}^{e} \cdot \boldsymbol{\sigma}
$$

with some coefficients $a^{e}$ and $\mathbf{b}^{e}$ and $e= \pm$ represents positive-/negative-energy states.

In the rest frame, the standard spinors $u$ and $v$ are given as [54]

$$
\begin{aligned}
& u(\mathbf{0},+)=\sqrt{2 m}(1,0,0,0)^{T}, \\
& u(\mathbf{0},-)=\sqrt{2 m}(0,1,0,0)^{T}, \\
& v(\mathbf{0},+)=\sqrt{2 m}(0,0,1,0)^{T}, \\
& v(\mathbf{0},-)=\sqrt{2 m}(0,0,0,1)^{T} .
\end{aligned}
$$

Note that $u(\mathbf{0},+)$ corresponds to a particle with spin parallel to the $z$-direction, while $v(\mathbf{0},+)$ corresponds to an antiparticle with spin antiparallel to the $z$-direction.

We diagonalize the distribution functions $f^{(0) e}$ in the rest frame,

$$
f_{s}^{(0) e} \delta_{r s}=\sum_{r^{\prime} s^{\prime}}\left(D^{e}\right)_{r r^{\prime}}^{\dagger} f_{r^{\prime} s^{\prime}}^{(0) e} D_{s^{\prime} s}^{e},
$$

with $D^{e}$ being $2 \times 2$ matrices in spin space,

$$
D^{e}=\left(\vec{d}_{+}^{e}, \vec{d}_{-}^{e}\right),
$$

where $\vec{d}_{ \pm}^{e}$ are the eigenvectors of $\mathbf{b}^{e} \cdot \boldsymbol{\sigma}$ corresponding to the eigenvalues \pm , respectively,

$$
\left(\mathbf{n}^{e} \cdot \boldsymbol{\sigma}\right) \vec{d}_{ \pm}^{e}= \pm e \vec{d}_{ \pm}^{e},
$$

where $\mathbf{n}^{e} \equiv \mathbf{b}^{e} / \sqrt{\mathbf{b}^{e} \cdot \mathbf{b}^{e}}$ is the unit vector along the direction of $\mathbf{b}^{e}$. Note that the distribution functions $f^{(0) e}$ in general depend on the space-time coordinates $x^{\mu}$, thus the transformation matrices $D^{e}$ as well as $\mathbf{n}^{e}$ are defined locally. We then define the following spinors, which can be derived by rotating the standard ones,

$\tilde{u}(x, \mathbf{0}, s) \equiv \sum_{s^{\prime}} u\left(\mathbf{0}, s^{\prime}\right) D_{s^{\prime} s}^{+}(x)=\sqrt{2 m}\left(\begin{array}{c}\vec{d}_{s}^{+} \\ \overrightarrow{0}\end{array}\right)$,
$\tilde{v}(x, \mathbf{0}, s) \equiv \sum_{s^{\prime}} v\left(\mathbf{0}, s^{\prime}\right) D_{s^{\prime} s}^{-}(x)=\sqrt{2 m}\left(\begin{array}{c}\overrightarrow{0} \\ \vec{d}_{s}^{-}\end{array}\right)$.

The spinors $\tilde{u}(x, \mathbf{0}, \pm)$ and $\tilde{v}(x, \mathbf{0}, \pm)$ now correspond to particles/antiparticles with spin parallel/antiparallel to $\mathbf{n}^{ \pm}$. Using Eqs. (A4) and (A7) we obtain

$$
\begin{aligned}
\sum_{r s} & \bar{u}(\mathbf{0}, s) \gamma^{\mu} \gamma^{5} u(\mathbf{0}, r) f_{r s}^{(0)+}(x, \mathbf{0}) \\
& =\sum_{r s} \overline{\tilde{u}}(x, \mathbf{0}, s) \gamma^{\mu} \gamma^{5} \tilde{u}(x, \mathbf{0}, r) f_{s}^{(0)+}(x, \mathbf{0}) \delta_{r s} \\
& =2 m \sum_{s} s\left(0, \mathbf{n}^{+}\right) f_{s}^{(0)+}(x, \mathbf{0}),
\end{aligned}
$$

and similarly for the $v$-spinors. Then, performing a Lorentz transformation we obtain 


$$
\begin{aligned}
& \sum_{r s} \bar{u}(\mathbf{p}, s) \gamma^{\mu} \gamma^{5} u(\mathbf{p}, r) f_{r s}^{(0)+}(x, \mathbf{p}) \\
& \quad=2 m \sum_{s} s n^{+\mu}\left(x, \mathbf{p}, \mathbf{n}^{+}\right) f_{s}^{(0)+}(x, \mathbf{p}),
\end{aligned}
$$

and similarly

$$
\begin{aligned}
\sum_{r s} & \bar{v}(-\mathbf{p}, s) \gamma^{\mu} \gamma^{5} v(-\mathbf{p}, r) f_{r s}^{(0)-}(x,-\mathbf{p}) \\
& =2 m \sum_{s} s n^{-\mu}\left(x,-\mathbf{p},-\mathbf{n}^{-}\right) f_{s}^{(0)-}(x,-\mathbf{p})
\end{aligned}
$$

where $n^{ \pm \mu}$ is given by Eq. (27). We rewrite the axial-vector current as

$$
\mathcal{A}_{\mu}^{(0)}=m n_{\mu}^{(0)} A^{(0)} \delta\left(p^{2}-m^{2}\right),
$$

where the vector $n_{\mu}^{(0)}(x, p)$ and the distribution function $A^{(0)}(x, p)$ are determined by Eqs. (26) and (30), respectively.

Furthermore, we define

$$
s \Sigma^{+\mu \nu}(x, \mathbf{p}) \equiv \frac{1}{2 m} \overline{\tilde{u}}(x, \mathbf{p}, s) \sigma^{\mu \nu} \tilde{u}(x, \mathbf{p}, s)
$$

and

$$
s \Sigma^{-\mu \nu}(x, \mathbf{p}) \equiv \frac{1}{2 m} \overline{\tilde{v}}(x,-\mathbf{p}, s) \sigma^{\mu \nu} \tilde{v}(x,-\mathbf{p}, s) .
$$

We have

$$
\Sigma^{ \pm \mu \nu}(x, \mathbf{p})=-\frac{1}{m} \epsilon^{\mu \nu \alpha \beta} p_{\alpha} n_{\beta}^{ \pm}(x, \mathbf{p}),
$$

which can be easily checked in the rest frame using the Dirac representation of the $\gamma$-matrices and $\left[\sigma_{i}, \sigma_{j}\right]=2 i \epsilon_{i j k} \sigma_{k}$. Defining

$$
\Sigma^{(0) \mu \nu}(x, p) \equiv \theta\left(p^{0}\right) \Sigma^{+\mu \nu}(x, \mathbf{p})-\theta\left(-p^{0}\right) \Sigma^{-\mu \nu}(x, \mathbf{p}),
$$

we obtain the tensor current $\mathcal{S}_{\mu \nu}^{(0)}$ as

$$
\begin{aligned}
\mathcal{S}_{\mu \nu}^{(0)}(x, p)= & \frac{1}{(2 \pi \hbar)^{3}} \delta\left(p^{2}-m^{2}\right) \\
& \times \sum_{s}\left[\theta\left(p^{0}\right) \overline{\tilde{u}}(x, \mathbf{p}, s) \sigma_{\mu \nu} \tilde{u}(x, \mathbf{p}, s) f_{s}^{(0)+}(x, \mathbf{p})\right. \\
& \left.-\theta\left(-p^{0}\right) \overline{\tilde{v}}(-\mathbf{p}, s) \sigma_{\mu \nu} \tilde{v}(-\mathbf{p}, s) f_{s}^{(0)-}(x,-\mathbf{p})\right] \\
= & m \Sigma_{\mu \nu}^{(0)}(x, p) \delta\left(p^{2}-m^{2}\right) A^{(0)}(x, p) .
\end{aligned}
$$

Using

$$
\begin{aligned}
\overline{\tilde{u}}(x, \mathbf{p}, s) \tilde{u}(x, \mathbf{p}, s) & =-\overline{\tilde{v}}(x,-\mathbf{p}, s) \tilde{v}(x,-\mathbf{p}, s)=2 m, \\
\tilde{\tilde{u}}(x, \mathbf{p}, s) \gamma^{5} \tilde{u}(x, \mathbf{p}, s) & =\overline{\tilde{v}}(x,-\mathbf{p}, s) \gamma^{5} \tilde{v}(x,-\mathbf{p}, s)=0, \\
\overline{\tilde{u}}(x, \mathbf{p}, s) \gamma^{\mu} \tilde{u}(x, \mathbf{p}, s) & =-\overline{\tilde{v}}(x,-\mathbf{p}, s) \gamma^{\mu} \tilde{v}(x,-\mathbf{p}, s)=2 p^{\mu},
\end{aligned}
$$

the calculation of $\mathcal{F}^{(0)}, \mathcal{P}^{(0)}$, and $\mathcal{V}_{\mu}^{(0)}$ is straightforward.

Finally, we stress that the diagonalization procedure for the distribution function described in this Appendix is in general possible also at higher order in $\hbar$, even though the exact form of the spinors is not known.

\section{APPENDIX B: REDUNDANCY OF EQS. (11)-(20)}

In this section we prove that Eqs. (11)-(20) are not independent from each other. Combining Eqs. (11), (15), (16), and (20), we derive

$$
\begin{aligned}
0= & \frac{\hbar}{2 m} \nabla_{\mu}(\Pi \cdot \mathcal{V}-m \mathcal{F})-\frac{1}{2 m} \Pi_{\mu}(\hbar \nabla \cdot \mathcal{V}) \\
& -\frac{1}{m} \Pi^{\nu}\left(\frac{\hbar}{2} \nabla_{[\mu} \mathcal{V}_{\nu]}-\epsilon_{\mu \nu \alpha \beta} \Pi^{\alpha} \mathcal{A}^{\beta}-m \mathcal{S}_{\mu \nu}\right) \\
& +\frac{\hbar}{2 m} \nabla^{\nu}\left(\Pi_{[\mu} \mathcal{V}_{\nu]}+\frac{\hbar}{2} \epsilon_{\mu \nu \alpha \beta} \nabla^{\alpha} \mathcal{A}^{\beta}\right)
\end{aligned}
$$

After some calculation we obtain

$$
\begin{aligned}
& \frac{\hbar}{2} \nabla_{\mu} \mathcal{F}+\Pi^{\nu} \mathcal{S}_{\nu \mu} \\
& =\frac{\hbar}{2 m}\left(\left[\nabla_{\mu}, \Pi_{\nu}\right]+\left[\nabla_{\nu}, \Pi_{\mu}\right]\right) \mathcal{V}^{\nu}+\frac{\hbar}{2 m}\left[\Pi^{\nu}, \nabla_{\nu}\right] \mathcal{V}_{\mu} \\
& \quad+\frac{1}{2 m} \epsilon_{\mu \nu \alpha \beta}\left(\left[\Pi^{\nu}, \Pi^{\alpha}\right]+\frac{\hbar^{2}}{4}\left[\nabla^{\nu}, \nabla^{\alpha}\right]\right) \mathcal{A}^{\beta} .
\end{aligned}
$$

The commutators can be easily calculated using the definition of the operators (7):

$$
\begin{aligned}
& {\left[\Pi_{\mu}, \Pi_{\nu}\right]=-\hbar j_{1}(\Delta) F_{\mu \nu}-\frac{\hbar}{2} \Delta j_{1}^{\prime}(\Delta) F_{\mu \nu},} \\
& {\left[\Pi_{\mu}, \nabla_{\nu}\right]=\Delta j_{1}(\Delta) F_{\mu \nu}-j_{0}(\Delta) F_{\mu \nu},} \\
& {\left[\nabla_{\mu}, \nabla_{\nu}\right]=\frac{2}{\hbar} \Delta j_{0}(\Delta) F_{\mu \nu},}
\end{aligned}
$$

where $j_{1}^{\prime}(x) \equiv \frac{d}{d x} j_{1}(x)$. Using the definitions of the spherical Bessel functions we can prove

$$
x j_{0}(x)-2 j_{1}(x)-x j_{1}^{\prime}(x)=0 .
$$

Inserting the commutators into Eq. (B2) and using the above relation, one finds that the right-hand side of Eq. (B2) vanishes, and we just obtain Eq. (18).

Analogously, we can construct the following equation using Eqs. (12), (15), (17), and (20), 


$$
\begin{aligned}
0= & \frac{1}{m} \Pi_{\mu}\left(\frac{\hbar}{2} \nabla \cdot \mathcal{A}+m \mathcal{P}\right)-\frac{\hbar}{2 m} \nabla_{\mu}(\Pi \cdot \mathcal{A})-\frac{\hbar}{4 m} \epsilon_{\mu \nu \alpha \beta} \\
& \times \nabla^{\nu}\left[\frac{\hbar}{2}\left(\nabla^{\alpha} \mathcal{V}^{\beta}-\nabla^{\beta} \mathcal{V}^{\alpha}\right)-\epsilon^{\alpha \beta \rho \sigma} \Pi_{\rho} \mathcal{A}_{\sigma}-m \mathcal{S}^{\alpha \beta}\right] \\
& -\frac{1}{2 m} \epsilon_{\mu \nu \alpha \beta} \Pi^{\nu}\left(\Pi^{\alpha} \mathcal{V}^{\beta}-\Pi^{\beta} \mathcal{V}^{\alpha}+\frac{\hbar}{2} \epsilon^{\alpha \beta \rho \sigma} \nabla_{\rho} \mathcal{A}_{\sigma}\right),
\end{aligned}
$$

from which we get

$$
\begin{aligned}
& \Pi_{\mu} \mathcal{P}+\frac{\hbar}{4} \epsilon_{\mu \nu \alpha \beta} \nabla^{\nu} \mathcal{S}^{\alpha \beta} \\
& =-\frac{\hbar}{2 m}\left(\left[\Pi_{\mu}, \nabla_{\nu}\right]+\left[\Pi_{\nu}, \nabla_{\mu}\right]\right) \mathcal{A}^{\nu}+\frac{\hbar}{2 m}\left[\Pi^{\nu}, \nabla_{\nu}\right] \mathcal{A}_{\mu} \\
& \quad+\frac{1}{2 m} \epsilon_{\mu \nu \alpha \beta}\left(\left[\Pi^{\nu}, \Pi^{\alpha}\right]+\frac{\hbar^{2}}{4}\left[\nabla^{\nu}, \nabla^{\alpha}\right]\right) \mathcal{V}^{\beta} .
\end{aligned}
$$

Analogously to Eq. (B2), the right-hand side of Eq. (B6) vanishes and we obtain Eq. (19).

\section{APPENDIX C: DERIVATION OF KINETIC EQUATIONS}

In this Appendix we show some technical details we used when deriving the kinetic equations (47), (49). First we focus on the kinetic equation for the zeroth-order dipole-moment tensor $\Sigma_{\mu \nu}^{(0)}$ and the axial distribution function $A^{(0)}$. We insert the vector part of Eq. (43) into Eq. (46) and use the relation $\mathcal{A}_{\mu}^{(0)}=-\frac{1}{2 m} \epsilon_{\mu \nu \alpha \beta} p^{\nu} \mathcal{S}^{\alpha \beta(0)}$, to derive

$$
\begin{aligned}
0= & -\frac{1}{2 m}\left[p_{\mu} \nabla^{(0) \alpha} \mathcal{S}_{\alpha \nu}^{(0)}-p_{\nu} \nabla^{(0) \alpha} \mathcal{S}_{\alpha \mu}^{(0)}\right] \\
& -\frac{1}{4 m} \epsilon_{\mu \nu \alpha \beta} \nabla^{(0) \alpha} \epsilon^{\beta \gamma \rho \sigma} p_{\gamma} \mathcal{S}_{\rho \sigma}^{(0)} \\
= & -\frac{1}{2 m}\left\{\left[\nabla^{(0) \alpha} p_{[\mu}\right] \mathcal{S}_{\nu] \alpha}^{(0)}+p^{\alpha} \nabla_{\alpha}^{(0)} \mathcal{S}_{\mu \nu}^{(0)}\right\} .
\end{aligned}
$$

Inserting the zeroth-order solution we get

$$
\delta\left(p^{2}-m^{2}\right)\left\{p \cdot \nabla^{(0)}\left[\Sigma_{\mu \nu}^{(0)} A^{(0)}\right]-F_{[\mu}^{\alpha} \Sigma_{\nu] \alpha}^{(0)} A^{(0)}\right\}=0 .
$$

The dipole-moment tensor is normalized, $\Sigma^{(0) \mu \nu} \Sigma_{\mu \nu}^{(0)}=2$, thus contracting the above equation with $\Sigma^{(0) \mu \nu}$ we obtain

$$
\delta\left(p^{2}-m^{2}\right) p \cdot \nabla^{(0)} A^{(0)}=0,
$$

where we have used

$$
\Sigma^{(0) \mu \nu}\left[F_{\mu}^{\alpha} \Sigma_{\nu \alpha}^{(0)}-F_{\nu}^{\alpha} \Sigma_{\mu \alpha}^{(0)}\right]=2 F_{\alpha \mu} \Sigma^{(0) \mu \nu} \Sigma_{\nu}^{(0) \alpha}=0,
$$

because $F_{\alpha \mu}$ is antisymmetric and $\Sigma^{(0) \mu \nu} \Sigma_{\nu}^{(0) \alpha}$ is symmetric under $\alpha \leftrightarrow \mu$. Inserting Eq. (C3) into Eq. (C2) one obtains the kinetic equation for $\Sigma_{\mu \nu}^{(0)}$.

The kinetic equation for $V^{(1)}$ is derived from the first line of Eq. (48). According to Eq. (43), $\mathcal{V}^{(1)}$ can be expressed in terms of $\mathcal{F}^{(1)}$ and $\mathcal{S}_{\mu \nu}^{(0)}$. Thus we get

$$
\frac{1}{m} p \cdot \nabla^{(0)} \mathcal{F}^{(1)}+\frac{1}{2 m} \nabla^{(0) \mu} \nabla^{(0) \nu} \mathcal{S}_{\mu \nu}^{(0)}=0 .
$$

The dipole-moment tensor is antisymmetric in its indices, so we can use the commutator $\left[\nabla^{(0) \mu}, \nabla^{(0) \nu}\right]=\left(\partial_{x}^{\alpha} F^{\mu \nu}\right) \partial_{p \alpha}$ to simplify the second term. Using also the zeroth- and firstorder solutions we obtain

$$
\begin{aligned}
0= & \frac{1}{m} p \cdot \nabla^{(0)} \mathcal{F}^{(1)}+\frac{1}{4 m}\left(\partial_{x}^{\alpha} F^{\mu \nu}\right) \partial_{p \alpha} \mathcal{S}_{\mu \nu}^{(0)} \\
= & \delta\left(p^{2}-m^{2}\right) p \cdot \nabla^{(0)} V^{(1)} \\
& -\frac{1}{2} \delta^{\prime}\left(p^{2}-m^{2}\right) p \cdot \nabla^{(0)}\left[F^{\alpha \beta} \Sigma_{\alpha \beta}^{(0)} A^{(0)}\right] \\
& +\frac{1}{4}\left(\partial_{x}^{\alpha} F^{\mu \nu}\right) \partial_{p \alpha}\left[\Sigma_{\mu \nu}^{(0)} A^{(0)} \delta\left(p^{2}-m^{2}\right)\right] \\
= & \delta\left(p^{2}-m^{2}\right)\left\{p \cdot \nabla^{(0)} V^{(1)}+\frac{1}{4}\left(\partial_{x}^{\alpha} F^{\mu \nu}\right) \partial_{p \alpha}\left[\Sigma_{\mu \nu}^{(0)} A^{(0)}\right]\right\} \\
& -\frac{1}{2} \delta^{\prime}\left(p^{2}-m^{2}\right) F^{\alpha \beta} p \cdot \nabla^{(0)}\left[\Sigma_{\alpha \beta}^{(0)} A^{(0)}\right] .
\end{aligned}
$$

In order to derive the kinetic equation for the first-order dipole-moment tensor, we first need $\mathcal{V}_{\mu}^{(2)}$, which is calculated by expanding Eq. (32) into a series in $\hbar$ and identifying the $\hbar^{2}$ term,

$$
\mathcal{V}_{\mu}^{(2)}=\frac{1}{m} p_{\mu} \mathcal{F}^{(2)}+\frac{1}{m} \Pi_{\mu}^{(2)} \mathcal{F}^{(0)}+\frac{1}{2 m} \nabla^{(0) \nu} \mathcal{S}_{\mu \nu}^{(1)} .
$$

Inserting this, as well as $\mathcal{A}_{\mu}^{(1)}$ from Eq. (43) into the second line of Eq. (48) we get

$$
\begin{aligned}
0= & \frac{1}{m}\left(\left[\Pi_{\mu}^{(2)}, p_{\nu}\right]-\left[\Pi_{\nu}^{(2)}, p_{\mu}\right]\right) \mathcal{F}^{(0)}-\frac{1}{2 m} p \cdot \nabla^{(0)} \mathcal{S}_{\mu \nu}^{(1)} \\
& +\frac{1}{2 m}\left(\left[\nabla^{(0) \alpha}, p_{\mu}\right] \mathcal{S}_{\alpha \nu}^{(1)}-\left[\nabla^{(0) \alpha}, p_{\nu}\right] \mathcal{S}_{\alpha \mu}^{(1)}\right) .
\end{aligned}
$$

The commutators are given by $\left[\nabla^{(0) \alpha}, p_{\mu}\right]=F_{\mu}{ }^{\alpha}$ and

$$
\left[\Pi_{\mu}^{(2)}, p_{\nu}\right]-\left[\Pi_{\nu}^{(2)}, p_{\mu}\right]=-\frac{1}{4}\left(\partial_{x \alpha} F_{\mu \nu}\right) \partial_{p}^{\alpha}
$$

Inserting the solutions for $\mathcal{F}^{(0)}$ and $\mathcal{S}_{\mu \nu}^{(1)}$ from Eqs. (37) and (41) into Eq. (C8) and using the above commutators, one obtains the kinetic equation for $\bar{\Sigma}_{\mu \nu}^{(1)}$. 
[1] F. Antinori, A. Dainese, P. Giubellino, V. Greco, M. P. Lombardo, and E. Scomparin, Nucl. Phys. A982, 1 (2019).

[2] A. Kurkela and A. Mazeliauskas, Phys. Rev. D 99, 054018 (2019).

[3] D. E. Kharzeev, L. D. McLerran, and H. J. Warringa, Nucl. Phys. A803, 227 (2008).

[4] D. T. Son and P. Surowka, Phys. Rev. Lett. 103, 191601 (2009).

[5] D. E. Kharzeev, J. Liao, S. A. Voloshin, and G. Wang, Prog. Part. Nucl. Phys. 88, 1 (2016).

[6] D. T. Son and N. Yamamoto, Phys. Rev. Lett. 109, 181602 (2012).

[7] M. A. Stephanov and Y. Yin, Phys. Rev. Lett. 109, 162001 (2012).

[8] D. T. Son and N. Yamamoto, Phys. Rev. D 87, 085016 (2013).

[9] J.-W. Chen, S. Pu, Q. Wang, and X.-N. Wang, Phys. Rev. Lett. 110, 262301 (2013).

[10] C. Manuel and J. M. Torres-Rincon, Phys. Rev. D 89, 096002 (2014).

[11] J.-Y. Chen, D. T. Son, M. A. Stephanov, H.-U. Yee, and Y. Yin, Phys. Rev. Lett. 113, 182302 (2014).

[12] C. Manuel and J. M. Torres-Rincon, Phys. Rev. D 90, 076007 (2014).

[13] J.-Y. Chen, D. T. Son, and M. A. Stephanov, Phys. Rev. Lett. 115, 021601 (2015).

[14] E. V. Gorbar, V. A. Miransky, I. A. Shovkovy, and P. O. Sukhachov, Phys. Rev. B 95, 205141 (2017).

[15] Y. Hidaka, S. Pu, and D.-L. Yang, Phys. Rev. D 95, 091901 (2017).

[16] Y. Hidaka, S. Pu, and D.-L. Yang, Phys. Rev. D 97, 016004 (2018).

[17] A. Huang, S. Shi, Y. Jiang, J. Liao, and P. Zhuang, Phys. Rev. D 98, 036010 (2018).

[18] J.-H. Gao, Z.-T. Liang, Q. Wang, and X.-N. Wang, Phys. Rev. D 98, 036019 (2018).

[19] D.-L. Yang, Phys. Rev. D 98, 076019 (2018).

[20] J.-h. Gao, J.-y. Pang, and Q. Wang, Phys. Rev. D 100, 016008 (2019).

[21] N. Mueller and R. Venugopalan, Phys. Rev. D 97, 051901 (2018).

[22] N. Mueller and R. Venugopalan, Phys. Rev. D 96, 016023 (2017).

[23] N. Mueller and R. Venugopalan, Phys. Rev. D 99, 056003 (2019).

[24] Y. Neiman and Y. Oz, J. High Energy Phys. 03 (2011) 023.

[25] M. V. Isachenkov and A. V. Sadofyev, Phys. Lett. B 697, 404 (2011).

[26] A. Einstein and W. de Haas, Dtsch. Phys. Ges., Verh. 17, 152 (1915) [Proc. KNAW 181, 696 (1915)].

[27] S. J. Barnett, Rev. Mod. Phys. 7, 129 (1935).

[28] L. Adamczyk et al. (STAR Collaboration), Nature (London) 548, 62 (2017).

[29] Z.-T. Liang and X.-N. Wang, Phys. Rev. Lett. 94, 102301 (2005); 96, 039901(E) (2006).

[30] J.-H. Gao, S.-W. Chen, W.-t. Deng, Z.-T. Liang, Q. Wang, and X.-N. Wang, Phys. Rev. C 77, 044902 (2008).
[31] S.-w. Chen, J. Deng, J.-h. Gao, and Q. Wang, Front. Phys. China 4, 509 (2009).

[32] F. Becattini, F. Piccinini, and J. Rizzo, Phys. Rev. C 77, 024906 (2008).

[33] F. Becattini, V. Chandra, L. Del Zanna, and E. Grossi, Ann. Phys. (Amsterdam) 338, 32 (2013).

[34] F. Becattini, L. Csernai, and D. J. Wang, Phys. Rev. C 88, 034905 (2013); 93, 069901(E) (2016).

[35] F. Becattini, I. Karpenko, M. Lisa, I. Upsal, and S. Voloshin, Phys. Rev. C 95, 054902 (2017).

[36] W. Florkowski, B. Friman, A. Jaiswal, and E. Speranza, Phys. Rev. C 97, 041901 (2018).

[37] W. Florkowski, B. Friman, A. Jaiswal, R. Ryblewski, and E. Speranza, Phys. Rev. D 97, 116017 (2018).

[38] W. Florkowski, E. Speranza, and F. Becattini, Acta Phys. Pol. B 49, 1409 (2018).

[39] W. Florkowski, A. Kumar, and R. Ryblewski, arXiv:1811.04409, 10.1016/j.ppnp.2019.07.001.

[40] F. Becattini, W. Florkowski, and E. Speranza, Phys. Lett. B 789, 419 (2019).

[41] R.-h. Fang, L.-g. Pang, Q. Wang, and X.-n. Wang, Phys. Rev. C 94, 024904 (2016).

[42] W. Florkowski, A. Kumar, and R. Ryblewski, Phys. Rev. C 98, 044906 (2018).

[43] U. W. Heinz, Phys. Rev. Lett. 51, 351 (1983).

[44] H. T. Elze, M. Gyulassy, and D. Vasak, Nucl. Phys. B276, 706 (1986).

[45] D. Vasak, M. Gyulassy, and H. T. Elze, Ann. Phys. (N.Y.) 173, 462 (1987).

[46] P. Zhuang and U. W. Heinz, Ann. Phys. (N.Y.) 245, 311 (1996).

[47] W. Florkowski, J. Hufner, S. P. Klevansky, and L. Neise, Ann. Phys. (N.Y.) 245, 445 (1996).

[48] J.-P. Blaizot and E. Iancu, Phys. Rep. 359, 355 (2002).

[49] Q. Wang, K. Redlich, H. Stoecker, and W. Greiner, Phys. Rev. Lett. 88, 132303 (2002).

[50] S. R. De Groot, W. A. Van Leeuwen, and C. G. Van Weert, Relativistic Kinetic Theory. Principles and Applications (North-Holland Amsterdam, 1980).

[51] I. Bailey and W. Israel, Commun. Math. Phys. 42, 65 (1975).

[52] I. Bialynicki-Birula, P. Gornicki, and J. Rafelski, Phys. Rev. D 44, 1825 (1991).

[53] V. Bargmann, L. Michel, and V. L. Telegdi, Phys. Rev. Lett. 2, 435 (1959).

[54] C. Itzykson and J.-B. Zuber, Quantum Field Theory (Courier Corporation, USA, 2012).

[55] M. Stone, V. Dwivedi, and T. Zhou, Phys. Rev. D 91, 025004 (2015).

[56] W. Israel, Gen. Relativ. Gravit. 9, 451 (1978).

[57] C. Cercignani and G. M. Kremer, The Relativistic Boltzmann Equation: Theory and Applications (Birkhäuser, Switzerland, 2002).

[58] F. Becattini, Phys. Rev. Lett. 108, 244502 (2012).

[59] S. Lin and L. Yang, Phys. Rev. D 98, 114022 (2018).

[60] G. S. Denicol, X.-G. Huang, E. Molnár, G. M. Monteiro, H. Niemi, J. Noronha, D. H. Rischke, and Q. Wang, Phys. Rev. D 98, 076009 (2018). 
[61] G. S. Denicol, E. Molnár, H. Niemi, and D. H. Rischke, Phys. Rev. D 99, 056017 (2019).

[62] J.-H. Gao and Z.-T. Liang, arXiv:1902.06510.

[63] K. Hattori, Y. Hidaka, and D.-L. Yang, arXiv:1903.01653.
[64] Z. Wang, X. Guo, S. Shi, and P. Zhuang, Phys. Rev. D 100, 014015 (2019).

[65] E. Leader, Spin in Particle Physics (Cambridge University Press, Cambridge, England, 2001). 